Aus der Abteilung Allgemeinmedizin

(Prof. Dr. med. M. M. Kochen, MPH, FRCGP)

im Zentrum Innere Medizin

der Medizinischen Fakultät der Universität Göttingen

\title{
Sexuelle Funktionsstörungen \\ bei Männern in der Hausarztpraxis
}

\author{
Inaugural - Dissertation \\ zur Erlangung des Doktorgrades \\ der Medizinischen Fakultät
}

der Georg-August-Universität zu Göttingen

\author{
vorgelegt von \\ Carmen Aschka \\ aus \\ Lingen (Ems)
}

Göttingen 2000 
Dekan: Prof. Dr. med. M. Droese

I. Berichterstatter: Prof. Dr. med. M.M. Kochen, MPH, FRCGP

II. Berichterstatter/in: Priv. Doz. Dr. med. G. Zöller

III. Berichterstatter/in: PD Dr. med. Ch. Hermann-Lingen

Tag der mündlichen Prüfung: 21. November 2002 
1. Einleitung 6

$\begin{array}{lll}\text { 2. Stand der Forschung } & 7\end{array}$

$\begin{array}{lll}2.1 & \text { Definition sexueller Funktionsstörungen } & 7\end{array}$

$2.2 \quad$ Epidemiologie 8

2.3 Ursachen 9

$\begin{array}{lll}2.4 \quad B e h a n d l u n g & 11\end{array}$

2.5 Sexuelle Dysfunktionen in der hausärztlichen Praxis 12

$\begin{array}{ll}3 . & 16\end{array}$

$\begin{array}{ll}\text { 4. } & 18\end{array}$

$\begin{array}{lll}4.1 & \text { Patientenfragebogen } & 18\end{array}$

$\begin{array}{lll}4.2 & \text { Arztfragebogen } & 19\end{array}$

$\begin{array}{lll}4.3 & \text { Pilotstudie } & 20\end{array}$

4.4 Auswahl der Arztpraxen 20

4.5 Auswahl der Patienten und Ablauf der Patientenbefragung 21

$\begin{array}{lll}4.6 & \text { Auswertung }\end{array}$

$\begin{array}{ll}\text { 5. Ergebnisse } 23 & 23\end{array}$

5.1 Reaktionen auf die Untersuchung 23

5.2 Ärzte 24

5.2.1 Stichprobe 24

5.2.2 Arztfragebogen 25

5.2.2.1 Häufigkeit von Sexualstörungen in der Hausarztpraxis 26

5.2.2.2 Ansprechen von Sexualproblemen durch den Hausarzt 26

5.2.2.3 Gründe für ein Nicht-Ansprechen sexueller Probleme 28 
5.2.2.4 Bedeutung sexualmedizinischer Kenntnisse und von Sexualberatung

5.2.2.5 Behandlung von Sexualproblemen in der Hausarztpraxis 30

5.2.2.6 Stellenwert von Sildenafil $\left(\right.$ Viagra $\left.^{\circledR}\right) \quad 31$

5.2.2.7 Fortbildungswünsche der Hausärzte 32

5.3 Patienten 32

5.3.1 Stichprobe 32

5.3.2 Konsultationsanlaß und Beziehung zum Hausarzt 35

5.3.3 Rolle des Hausarztes aus Patientensicht 36

5.3.4 Häufigkeit, Ausprägung und Ursachen sexueller Probleme 37

5.3.5 Betreuung bei Sexualproblemen durch den Hausarzt 42

5.3.6 Wünsche an den Hausarzt 43

5.4 Einflüsse auf das Antwortverhalten 44

5.4.1 Alter der Patienten 44

$\begin{array}{lll}\text { 5.4.2 Behandlungsdauer } & 47\end{array}$

$\begin{array}{lll}\text { 5.4.3 Persönliche Lebensumstände } & 47\end{array}$

5.4.4 Sexualstörungen und sexuelle Zufriedenheit 48

5.4.5 Einschätzung sexualmedizinischer Kenntnisse durch
den Hausarzt

$\begin{array}{lll}\text { 5.4.6 Wohnort } & 50\end{array}$

6. Diskussion 51

6.1 Zielsetzung der Studie 51

6.2 Beurteilung der Methode 51

6.3 Die Stichprobe 52

6.4 Häufigkeit von Sexualproblemen in der hausärztlichen Praxis 53

6.5 Gespräche über Sexualprobleme und mögliche Barrieren 54

6.6 Einflußfaktoren auf die Gesprächsbereitschaft 57

6.7 Medizinische Bedeutung der Sexualanamnese 58

6.8 Viagra $^{\circledR}$ in der Hausarztpraxis $\quad 59$

6.9 Ausblick 61 
$\begin{array}{ll}\text { 7. Zusammenfassung } & 62\end{array}$

8. Anhang 63

8.1 Arztfragebogen 64

8.2 Arztfragebogen (Grundauszählung) 67

$\begin{array}{lll}8.3 & \text { Patientenfragebogen } & 72\end{array}$

8.4 Patientenfragebogen (Grundauszählung) 76

$\begin{array}{ll}\text { 9. Literaturverzeichnis } & 81\end{array}$

Anmerkung:

Der besseren Lesbarkeit wegen wird im Text die männliche Sprachform gewählt. Selbstverständlich sind beide Geschlechter gemeint. 
Abbildung 1: Wie häufig initiieren Hausärzte ein Gespräch bei möglichen Sexualstörungen?

Abbildung 2: Wie häufig werden Partner in die Behandlung sexueller Funktionsstörungen einbezogen?

Abbildung 3: Die Bedeutung des Geschlechts des Hausarztes für Gespräche über Sexualität

Tabelle 2: Anlässe für Gespräche über mögliche Sexualprobleme

Tabelle 3: Gespräche über Sexualität bei Vorliegen bestimmter Krankheiten

Tabelle 4: Gründe für Nicht-Ansprechen sexueller Probleme aus Sicht des Arztes

Tabelle 5: Bedeutung von sexualmedizinischen Kenntnissen und von Sexualberatung in der Hausarztpraxis aus Sicht des Arztes

Tabelle 6: Beurteilung der eigenen sexualmedizinischen Kenntnisse

Tabelle 7: Behandlung sexueller Störungen in der Hausarztpraxis 
Tabelle 9: Alter der teilnehmenden Patienten

Tabelle 10: Familienstand, Wohnort und Beruf der befragten Patienten

Tabelle 11: Chronische Krankheiten

Tabelle 12: Konsultationsanlaß

Tabelle 13: Kenntnis des Hausarztes über die persönlichen Lebensumstände

Tabelle 14: Häufigkeit und Ausprägung sexueller Probleme

Tabelle 15: Ursachen der Sexualprobleme

Tabelle 16: Auswirkungen der Sexualstörungen

Tabelle 17: Mögliche Ansprechpartner bei Problemen im sexuellen Bereich

Tabelle 18: Wünsche an den Hausarzt

Tabelle 19: Hausarzt soll Sexualprobleme während einer Behandlung direkt ansprechen

Tabelle 20: Bedeutung des Geschlechts des Hausarztes als potentieller Gesprächspartner bei sexuellen Problemen

Tabelle 21: Sexualprobleme in Abhängigkeit vom Alter

Tabelle 22: Ursachen in Abhängigkeit vom Alter

Tabelle 23: Gesprächsbereitschaft der Patienten und Kenntnis des Hausarztes über die persönlichen Lebensumstände

Tabelle 24: Zufriedenheit mit dem Sexualleben in Abhängigkeit von dem Vorliegen sexueller Schwierigkeiten 


\section{Einleitung}

Sexuelle Funktionsstörungen und Sexualprobleme sind in der männlichen Bevölkerung weit verbreitet. Sie können auf organische und/oder psychische Ursachen zurückgeführt werden; sexuelle Dysfunktionen können ihrerseits organische und/oder psychische Beschwerden zur Folge haben.

Obwohl das Thema Sexualität immer noch tabuisiert ist, erscheint gerade der Hausarzt als idealer Ansprechpartner für Patienten mit Sexualproblemen, denn er ist mit ihrer persönlichen und familiären Situation meist seit vielen Jahren vertraut. Überdies stehen sexuelle Funktionsstörungen oft im Zusammenhang mit Krankheiten bzw. Medikamenten, die der Hausarzt im Regelfall kennt. Dennoch werden sexuelle Fragen und Probleme in der hausärztlichen Praxis selten thematisiert.

In Deutschland liegen hierzu keine Untersuchungen vor. Ziel dieser Arbeit war es, den hausärztlichen Betreuungsbedarf von Männern mit Sexualstörungen wie auch mögliche Hindernisse bei der Betreuung in Erfahrung zu bringen. Verglichen wurden hierbei die Arzt- und Patientenperspektiven.

Der Stand der Forschung wird in Kapitel 2 referiert. In Kapitel 3 werden die Arbeitshypothesen vorgestellt. Praxen- und Patientenwahl sowie die Untersuchungsmethoden sind in Kapitel 4 beschrieben. Kapitel 5 und 6 enthalten die Ergebnisse und Diskussion. Zusammenfassung (Kapitel 7), Anhang (Kapitel 8, enthält die Originalfragebögen mit Grundauszählungen) sowie Literaturverzeichnis (Kapitel 9) beschließen die Arbeit. 


\section{Stand der Forschung}

\subsection{Definition}

Als sexuelle Dysfunktion oder sexuelle Funktionsstörungen werden Störungen im Ablauf des sexuellen Reaktionszyklus bezeichnet, die von den Betroffenen (bzw. von ihren Partnern) als nachteilig empfunden werden (Pschyrembel 1998). Die meisten Untersuchungen zur sexuellen Dysfunktion bei Männern beziehen sich auf erektile Dysfunktion. Dieser Begriff löst im wissenschaftlichen Bereich zunehmend den Begriff der Impotenz ab, um negative Assoziationen zu vermeiden. Folgende Formen sexueller Funktionsstörungen werden unterschieden:

a) Appetenzstörung = Libidostörung = Lustlosigkeit

der fehlende oder verminderte Versuch, sexuelle Aktivität aufzunehmen (Kockott 1988)

b) Erektile Dysfunktion = Impotenz

die Unfähigkeit des Mannes, eine Erektion für einen befriedigenden Geschlechtsverkehr zu erreichen oder aufrechtzuerhalten (Suter 1998)

c) Störungen der Ejakulation

- Ejaculatio praecox = vorzeitiger Samenerguß

die unbefriedigende Kontrolle des Mannes über den Zeitpunkt seines Orgasmus (Pschyrembel 1998)

- Ejaculatio retardata = verzögerter Samenerguß

Ejakulation und Orgasmus werden subjektiv als zu spät eintretend empfunden (Pschyrembel 1998)

- Retrograde Ejakulation

Ejakulation in die Harnblase bei ungestörtem Orgasmuserleben (Kockott 1988), bedingt durch mangelnden Verschluß des Blasenhalses 


\subsection{Epidemiologie}

Die Angaben zur Prävalenz sexueller Funktionsstörungen schwanken stark. Eine US-amerikanische Studie mit 1410 Männern zwischen 18 und 59 Jahren fand eine sexuelle Dysfunktion bei 31 \% der Männer (Laumann et al. 1999). In dieser Studie wurden die Männer u.a. zu Sexualfragen interviewt. Fragebogen-Untersuchungen bei hausärztlichen Patienten in England, Israel und den USA stellten eine Prävalenz sexueller Funktionsstörungen bei Männern von 34 bis 56 \% fest (Dunn et al. 1998; Moore und Goldstein 1980; Read et al. 1997; Shahar et al. 1991). Wurde nach Kommunikationsproblemen mit dem Partner über sexuelle Fragen oder Unzufriedenheit mit der Frequenz sexueller Kontakte gefragt, so ergab sich eine Häufigkeit dieser Schwierigkeiten von 75 \% (Read et al. 1997). Hierbei konnten die Patienten ihre Probleme differenziert nach der Häufigkeit inres Auftretens angeben, so daß in die Prävalenz auch „seltene“ Probleme einfließen. Fast alle (97\%) der von Metz und Seifert (1990) befragten Männer hatten zu „irgendeinem Zeitpunkt im Leben“ ein sexuelles Problem.

Unter erektiler Dysfunktion im engeren Sinne leiden in Deutschland schätzungsweise 3 bis 7 Mio. Männer (Ahlen und Hertle 1996). Genaue Angaben liegen nicht vor. Zur Berechnung bzw. Schätzung der Prävalenz von erektiler Dysfunktion wird meist die 1994 mit Männern im Alter von 40 bis 70 Jahren durchgeführte Massachusetts Male Aging Study (Feldman et al. 1994) zu Grunde gelegt. Gelegentliche Erektionsstörungen kommen bei $35 \%$ der Männer vor, permanente hingegen nur bei 1,6 \% (Rönsberg 1998).

Für die Ejaculatio praecox reichen die Schätzungen in Deutschland von 4 \% (Rönsberg 1998) bis $28 \%$ (Buddeberg 1996). Angloamerikanische Untersuchungen berichten Prävalenzen von 14 bis $25 \%$ bei hausärztlichen Patienten (Dunn et al. 1998; Read et al. 1997; Shahar et al. 1991).

Der Zusammenhang zwischen dem Lebensalter und erektiler Dysfunktion ist eindeutig (Feldman et al. 1994). 52 \% der Männer zwischen 40 und 70 Jahren litten unter erektiler Dysfunktion. Mit zunehmendem Lebensalter stieg sowohl die Schwe- 
re erektiler Dysfunktion als auch der Anteil der betroffenen Männer an. Nach Laumann et al. (1999) hatten $18 \%$ der von innen untersuchten 50 bis 60-jährigen Männer Erektionsstörungen, verglichen mit $7 \%$ der unter 30-jährigen. Diesen Zusammenhang fanden auch Dunn et al. (1999) und Pinnock et al. (1999). Die Häufigkeit von Appetenzstörungen steigt ebenfalls deutlich mit zunehmendem Alter: von $4 \%$ bei 55-jährigen bis zu 58 \% bei 75-jährigen Männer (Laumann et al. 1999; Rönsberg 1998). Dieser Zusammenhang gilt nicht für alle Sexualstörungen (Ende et al. 1984). Die Ejaculatio praecox ist vor allem ein Problem jüngerer Männer (Rönsberg 1998).

Laumann et al. (1999) fanden, daß Männer und Frauen mit höherem Bildungsstand seltener über sexuelle Probleme berichteten. Im Vergleich zu Männern mit geringem Bildungsstand nannten sie z.B. seltener Ejaculatio praecox und allgemeine sexuelle Sorgen, dafür häufiger angenehme sexuelle Erfahrungen.

\subsection{Ursachen}

Sexuelle Probleme lassen sich auf organische und/oder psychische Ursachen zurückführen; eine Verknüpfung beider Faktoren ist häufig (Porst 1991).

Zu den psychischen Ursachen gehören Partnerprobleme, Ängste, Depressionen und Streß (Kockott 1988). Auch ein zu hoher Maßstab, der an eine „angemessene sexuelle Funktion" angelegt wird, kann Ursache sein (Tiefer 1993). Halvorsen und Metz (1991) teilen die psychischen Ursachen der sexuellen Dysfunktion in drei Kategorien ein:

1. Allgemeine psychische Faktoren (z.B. Depressionen, Angst, vermindertes Selbstbewußtsein, Schuldgefühle)

2. Beziehungsprobleme

3. Psychosexuelle Faktoren (z.B. Versagensängste oder sexuelle Traumata).

Dunn et al. (1999) fanden beispielsweise, daß der vorzeitige Samenerguß häufig mit Ängstlichkeit und Sorgen der betroffenen Patienten assoziiert ist. Sexuelle Funktionsstörungen werden häufig von psychosozialen Faktoren und emotionalen 
wie streßabhängigen Problemen beeinflußt (Laumann et al. 1999). Nach Senger und Hoffmann (1996) war die Schwere sexueller Störungen bei Männern positiv mit dem Grad der „Emanzipation“ der jeweiligen Partnerinnen assoziiert; d.h., je "emanzipierter" sich die befragten Frauen einschätzten, desto ausgeprägter waren die Sexualprobleme der jeweiligen Männer.

Organische Gründe für sexuelle Dysfunktionen umfassen lokale körperliche Schäden, vaskuläre Erkrankungen sowie endokrinologische und neurologische Störungen. Auch Leber- und Nierenerkrankungen können über Stoffwechselstörungen der Sexualhormone für Beeinträchtigungen im Sexualleben verantwortlich sein (Kockott 1988). Stärkster Risikofaktor für eine erektile Dysfunktion ist nach Feldman et al. (1994) ein Diabetes mellitus. Weitere Risikofaktoren für sexuelle Funktionsstörungen sind Hypercholesterinämie, Hypertonie und Nikotinabusus (Pinnock et al. 1999; Suter 1998), - Parameter einer Gefäßveränderung (Arteriosklerose). Nach Dunn et al. (1999) berichten Männer mit erektiler Dysfunktion häufig, unter Prostatabeschwerden, Diabetes mellitus oder Hypertonie zu leiden. Außerdem kann eine Vielzahl von Medikamenten Libido-, Erektions- oder Ejakulationsstörungen hervorrufen: z.B. bestimmte Antihypertonika, Diuretika und Psychopharmaka, speziell Antidepressiva (Baier und Phillip 1994; Finger et al. 1997; Müller-Oerlinghausen et al. 1999; Porst 1991).

Aufgrund verbesserter diagnostischer Möglichkeiten hat sich in den letzten Jahren das Verhältnis psychogener/organischer Ursachen bei Erektionsstörungen zu Gunsten organischer Ursachen verschoben, während diese vorher stärker als psychisch oder altersphysiologisch bedingt erklärt wurden (Foreman und Doherty 1993). Pathogenetisch bedeutsame organische Störungen sind bei 50 bis $80 \%$ der Männer mit Erektionsproblemen zu finden (Ahlen und Hertle 1996). Das schließt jedoch die Beteiligung psychogener Faktoren nicht aus: So können z.B. Versagensängste bei Patienten mit Diabetes mellitus (aufgrund des Wissens um den Risikofaktor, den diese Krankheit darstellt) Erektionsstörungen sowohl bedingen als auch aufrechterhalten. Selbst wenn Sexualprobleme nicht primär psychisch verursacht wurden, können psychische Probleme zusätzlich entstehen (Baker 1991; Beutel 1999; Miller 
2000; Utiger 1998): So berichteten Patienten mit erektiler Dysfunktion und Ejaculatio praecox von einer geminderten Lebensqualität (McCabe 1997). Auch führten die sexuellen Probleme zu Distanz in der Partnerschaft sowie zu geringeren sozialen Aktivitäten. Eine Studie aus den Niederlanden (Broekman et al. 1994a) zeigte, daß Patienten mit Erektionsstörungen als Grund für ihre Störung überwiegend organische Ursachen angaben, während ihre Hausärzte eher psychische Gründe vermuteten.

\subsection{Behandlung}

Bei organisch bedingten sexuellen Störungen kann gegebenenfalls die Ursache durch Medikationsänderung, Hormonsubstitution oder operative Korrektur von Fehlbildungen des Penis beseitigt werden. Auch eine Kompensation organischer Störungen ist möglich. So kann eine Erektion mittels Schwellkörperinjektion mit vasoaktiven Substanzen oder Einbringen des vasoaktiven Wirkstoffes Alprostadil in die Harnröhre, Vakuumapparaten oder Penisprothesen erreicht werden (Nehra et al. 1999; Porst 1992; Schaudig 1998; Sigusch 1995).

Seit Oktober 1998 besteht in Deutschland durch Sildenafil $\left(\right.$ Viagra $\left.^{\circledR}\right)$ die Möglichkeit, erektile Dysfunktion durch ein oral zu verabreichendes, wirkungsvolles und gut verträgliches Medikament zu therapieren (Goldstein et al. 1998; Morales et al. 1998). Der Wirkstoff Sildenafil bewirkt über die Blockade der Phosphordiesterase Typ 5, die sich im Penisschwellkörper befindet, einen Anstieg von cGMP, das wiederum die arterielle Gefäßmuskulatur erschlaffen läßt und somit zur Versteifung des Penis führt (Goldstein et al. 1998). Mögliche Nebenwirkungen sind unter anderem Kopfschmerzen, Diarrhoe und Verschiebungen der visuellen Wahrnehmung im blau/grün-Bereich sowie erhöhte Lichtempfindlichkeit. Männer, die Medikamente mit organischen Nitraten (z.B. bestimmte Herzmedikamente) einnehmen, sollten Sildenafil meiden, da diese Kombination den Blutdruck zu stark senken kann. Von Todesfällen im Zusammenhang mit der Einnahme von Viagra wurde berichtet (Kloner und Jarow 1999). Fraglich ist, ob dies auf das Medikament oder auf „ungewohnte Überanstrengung" bei Vorliegen von Herzkrankheiten zurückzuführen ist. 
Durch die vergleichsweise einfache Behandlung mit Sildenafil können mögliche psychische Komponenten als (Mit-)Ursachen für die erektile Dysfunktion in den Hintergrund treten. Einige Autoren führen an, daß durch Sildenafil die Diagnostik einer erektilen Dysfunktion eingeschränkt werden kann (Utiger 1998), weil möglicherweise nur noch "schwierige Fälle" an Spezialisten überwiesen werden (Hengeveld 1998). Schaudig (1999) merkt an, daß auch die Bereitschaft der Patienten, sich vor der Verschreibung eingehend untersuchen zu lassen, im Vergleich zu der „Zeit vor Viagra ${ }^{\circledR \text { « }}$ deutlich zurückgegangen ist. Andererseits suchen gerade durch Sildenafil und die dadurch bedingte öffentliche Präsenz des Themas Erektionsstörungen viele Männer mit Potenzproblemen, die vorher keine Hilfe suchten, ihren Arzt auf (Mobley und Baum 1998). In Deutschland hat sich die Zahl der Hilfe suchenden Männer bei erektiler Dysfunktion ein Jahr nach der Zulassung von Viagra ${ }^{\circledR}$ verdoppelt (Berghof 2000). Sildenafil ist in dieser Zeit hierzulande 500000 Männern verschrieben wo rden.

Ist eine Psychogenese gesichert, sollte ein sexualtherapeutisch geschulter Spezialist die Behandlung übernehmen oder ergänzen (Suter 1998). Für die Sexualtherapie ist die Teilnahme beider Partner Voraussetzung (Buddeberg 1994). Ziel der Behandlung ist es, die eigene Körperwahrnehmung und die Verständigung zwischen den Partnern zu verbessern sowie sexuelle Ängste schrittweise abzubauen.

Weil Organo-und Psychogenese nur schwer zu trennen sind, empfehlen einige Autoren eine „kombinierte“ Behandlung sexueller Dysfunktionen. Diese soll sowohl möglichen körperlichen Ursachen als auch psychischen Faktoren und/oder Folgen Rechnung tragen (Hartmann 1998; Riley und Athanasiadis 1997; Tiefer und SchützMüller 1995).

\subsection{Sexuelle Dysfunktionen in der hausärztlichen Praxis}

Familienmedizin umfaßt die somatische, psychische und soziale Betreuung von Familien oder familienähnlichen Gruppen (Ewert 1998). Bei der Vielschichtigkeit sexueller Funktionsstörungen bietet sich somit gerade der Hausarzt als erster An- 
sprechpartner an. Er ist meist mit der persönlichen und familiären Situation des Patienten seit vielen Jahren vertraut (Halvorsen und Metz 1991; Himmel und Kochen 1998; Rakel 1995) - eine Voraussetzung, die ein Gespräch über Sexualität đleichtert. Für die Therapie sexueller Funktionsstörungen kann es hilfreich sein, daß häufig beide Partner beim selben Hausarzt Patienten sind. Bei Bedarf können daher die Partner in die Behandlung mit einbezogen werden, zumal bei Sexualstörungen oftmals auch Beziehungskonflikte bestehen (Hartmann 1998; Kuritzky et al. 1998; Loewit 1996) und ein Gespräch mit dem Sexualpartner des Patienten die Diagnose erleichtern kann (Beutel 1999; Miller 2000).

Ob Männer bei Sexualproblemen lieber einen Arzt oder eine Ärztin konsultieren, ist nach Britt et al. (1996) differenziert zu beantworten: Bei Fragen zum männlichen Genitale wurden Ärzte häufiger aufgesucht als Ärztinnen. Andererseits wurden Ärztinnen eher zu psychosozialen Problemen zu Rate gezogen, so daß sich bei innen mehr Beratungen zur Familienplanung ergaben als bei ihren männlichen Kollegen.

Eine Sexualanamnese beinhaltet Fragen nach der Häufigkeit und Variabilität sexueller Aktivität sowie hierbei auftretenden Störungen und Problemen (Rönsberg 1998). Außerdem wird auf Bewertung, Moralvorstellungen und Ängste in bezug auf Sexualität eingegangen. Beim Mann läßt sich die Sexualanamnese an Fragen zum Urogenitalbereich anschließen (Dahmer 1994). Durch Gespräche über Sexualität erhalten Hausärzte in vielen Fällen Informationen von medizinischer Relevanz (Ende et al. 1984). Sexuelle Dysfunktion kann unter Umständen auf bisher nicht diagnostizierte Krankheiten wie Diabetes mellitus oder Arteriosklerose hinweisen. Andererseits können Sexualprobleme zu Schlaflosigkeit und Depressionen führen (Baker 1991; Shabsigh et al. 1998). Nach Schilling et al. (1999) besteht zudem ein Zusammenhang zwischen sexueller Zufriedenheit und körperlichen Beschwerden: Männer mit sexueller Unzufriedenheit äußerten im Vergleich zu einer altersgleichen Stichprobe signifikant häufiger und intensivere körperliche Beschwerden. Anamnestisch bedeutsam ist die Medikation, da Medikamente zu den häufigsten Gründen sexueller Störungen zählen (Holzapfel 1993). Beispielsweise werden die unerwünschten Wirkungen von Antidepressiva auf die Sexualität oft unterschätzt, obwohl sie die Compliance der Patienten erheblich beeinträchtigen können (Baier und 
Phillipp 1994; Müller-Oerlinghausen et al. 1999). Dies gilt ebenfalls für andere hausärztlich verordnete Dauermedikamente (z.B. Beta-Blocker).

Eine Sexualanamnese ist aber laut Buddeberg (1994) in der Praxis offensichtlich nicht die Regel: etwa jeder vierte Patient, der den Allgemeinarzt wegen gesundheitlicher Probleme aufsucht, leidet an einer länger dauernden sexuellen Funktionsstörung. Jedoch nur ungefähr $4 \%$ aller Patienten suchen ihren Arzt primär wegen sexueller Schwierigkeiten auf. Selbst bei vermuteter erektiler Dysfunktion beginnen Ärzte oftmals nicht von sich aus ein Gespräch darüber (Broekman et al. 1994b), obwohl sich Patienten dies wünschten (Metz und Seifert 1990; Shahar et al. 1991). Nach Perttula (1999) sprechen Ärzte selbst Risikopatienten nur selten auf mögliche Erektionsstörungen an. Gründe für diese Zurückhaltung könnten sein: Vernachlässigung dieses Themas im Studium (Sanders 1993), mangelndes Fachwissen sowie Zeitdruck (Broekman et al. 1994b), Schamgefühle und geringes Selbstbewußtsein (Merril et al. 1990). Sexualität ist immer noch ein Tabu-Thema - ähnlich wie Tod und Sterben (Holzapfel 1993). Levine sieht bei Ärzten eine unterschwellige Angst vor der eigenen Sexualität (1992). Nicht nur Ärzte, auch viele Patienten sind sich nicht darüber im klaren, daß Sexualprobleme ein „legitimes“ medizinisches Thema sind (Burnett 1998; Sanders 1993). Sie schweigen daher aus Scham (Buddeberg 1994). Diese Ängste und Unsicherheiten auf Arzt- und Patientenseite wurden von Ittner et al. (1997a,b) bei ihren Untersuchungen über ungewollte Kinderlosigkeit bestätigt. Nach Corney (1990) können zudem männliche Patienten persönliche Informationen nicht so leicht mitteilen wie Frauen. Sie scheinen die Suche nach Hilfe als „unmännlich“ zu empfinden und beschränken sich daher auf spezifische (nichtsexuelle) Gesundheitsprobleme in der Konsultation (Tudiver und Talbot 1999).

Bereits durch Gespräche und Informationen zur normalen Sexualphysiologie kann der Hausarzt betroffenen Patienten oftmals helfen (Hartmann 1998; Holzapfel 1993; Moore und Goldstein 1980). Der Hinweis auf die enorme Variationsbreite menschlichen Sexualverhaltens (Kockott 1989) kann manchmal eventuell vorhandene Ängste und Hemmungen abbauen oder Fehlvorstellungen und übersteigerte Erwartungen korrigieren. 
Zur Therapie sexueller Funktionsstörungen stehen dem Hausarzt aber auch Sildenafil, Hormontherapie und eventuell Schwellkörperinjektion bzw. AlprostadilApplikation sowie Vakuumapparate zur Verfügung. Gerade durch die Einführung von Sildenafil könnte der Hausarzt in Zukunft ein wichtiger Ansprechpartner in sexualmedizinischen Fragen werden (Tiefer 1998). Zwei Drittel der Hausärzte verschrieben ein Jahr nach der Zulassung von Viagra ${ }^{\circledR}$ das Medikament (Berghof 2000). Läßt sich das Problem in der Hausarztpraxis nicht lösen, empfiehlt sich in Abstimmung mit dem Patienten eine Überweisung an Spezialisten (Loewit 1996; Suter 1998). 


\section{Untersuchungshypothesen}

Angesichts der hohen (Lebenszeit-)Prävalenz sexueller Funktionsstörungen, der medizinischen Relevanz einer Sexualanamnese sowie der besonderen Rahmenbedingungen in der hausärztlichen Praxis bietet sich eine (Mit-)Betreuung von Männern mit Sexualproblemen durch den Hausarzt an. Ziel dieser Studie war es, den Bedarf von Patienten mit sexuellen Problemen wie auch die Möglichkeiten und Grenzen hausärztlicher Betreuung bei diesen Problemen in Erfahrung zu bringen. Insbesondere sollte herausgefunden werden, warum eine Sexualanamnese in der hausärztlichen Praxis selten erhoben wird. Im Hinblick auf die Zielsetzung der Arbeit wurden folgende Hypothesen formuliert:

\section{Patientenbezogene Hypothesen:}

1. Patienten möchten mit ihrem Hausarzt über Sexualität und bei Bedarf auch über sexuelle Probleme sprechen.

2. Patienten würden es begrüßen, wenn die Initiative, über Sexualität und Sexualprobleme zu sprechen, von Ihrem Hausarzt ausginge.

3. Etwa ein Drittel der Patienten in der hausärztlichen Praxis leidet zumindest gelegentlich unter Sexualproblemen.

4. Nur ein geringer Teil der Patienten, die unter Sexualstörungen leiden, sucht tatsächlich deshalb den Hausarzt auf.

5. Für Patienten ist es einfacher, mit ihrem Hausarzt über eventuell bestehende Sexualprobleme zu sprechen, wenn der Arzt sie und ihr soziales Umfeld schon seit längerem kennt.

6. Für den Großteil der Patienten spielt das Geschlecht des Hausarztes keine Rolle bei Gesprächen über Sexualprobleme. 


\section{Hausarztbezogene Hypothesen:}

7. Nach Meinung von Hausärzten stehen Sexualstörungen oft im Zusammenhang mit Krankheiten und Medikamenten, die häufig in der Hausarztpraxis vorkommen.

8. Sexualstörungen machen nach Meinung eines Großteils der Hausärzte eine Änderung der Medikation anderer Krankheiten notwendig.

9. Hausärzte erheben selten Sexualanamnesen in ihrer Praxis.

10. Hausärzte halten sexualmedizinische Kenntnisse und Sexualberatung für wichtig, schätzen die eigenen sexualmedizinischen Kenntnisse jedoch eher gering ein.

11. Neben geringem sexualmedizinischen Wissen und zeitlichen Gründen spielen für Hausärzte auch Schamgefühle eine Rolle, wenn sie Sexualprobleme nicht ansprechen.

12. Die Diskussion um Sildenafil $\left(\right.$ Viagra $\left.^{\circledR}\right)$ hat nach Erfahrung von Hausärzten zu häufigeren Beratungsanlässen zum Thema Sexualstörungen geführt. 


\section{Material und Methoden}

Da der Bereich sexueller Probleme oft noch tabuisiert ist, erschien eine Befragung der Patienten per Fragebogen geeigneter als ein persönliches Interview. Auf die Gewährleistung der Anonymität wurde besonders geachtet. Das „Werben“ der Patienten für die Studie sowie das Ausfüllen der Bögen fanden daher nicht im Wartezimmer, sondern in einem separaten Raum der jeweiligen Hausarztpraxis statt. Dort befand sich eine „Box“ für die ausgefüllten Fragebögen, die direkt an die Abteilung Allgemeinmedizin der Universität Göttingen weitergeleitet wurden. Weder Hausärzte noch Arzthelferinnen hatten Zugriff zu den Fragebögen.

Die Hausärzte wurden ebenfalls schriftlich befragt. Dies bedeutete einen relativ geringen Zeitaufwand für den Arzt, störte nicht den Praxisablauf und sollte sich daher positiv auf die Teilnahmequote auswirken.

\subsection{Patientenfragebogen}

Der Patientenfragebogen (s. Anhang) enthielt neben soziodemographischen Angaben Fragen zu folgenden Bereichen:

- Aktueller Konsultationsanlaß

- Möglichkeit zum Gespräch mit dem Hausarzt über persönliche Belange

- Beziehung zum Hausarzt

- Wünsche an den Hausarzt

- Häufigkeit, Ausprägung, Ursachen und Auswirkungen sexueller Probleme

- Behandlungen/Beratungen bei sexuellen Problemen durch den Hausarzt. 
Im Patientenfragebogen konnten die Männer auf einer Skala von 1 (sehr unzufrieden) bis 10 (sehr zufrieden) ihre sexuelle Zufriedenheit angeben. Für einige Auswertungen wurden die Skalen zur besseren Anschaulichkeit in drei Kategorien unterteilt:

- unzufrieden $(=1-3)$

- zufrieden (=4-7)

- $\quad$ sehr zufrieden $(=8-10)$.

Für die Untersuchung des möglichen Einflusses des Alters wurden die Patienten gegebenenfalls in zwei Altersgruppen eingeteilt $(<=45$ Jahre; $>45$ Jahre). Um den möglichen Einfluß der Behandlungsdauer zu klären, wurden die Patienten ebenfalls in zwei Gruppen unterteilt ( $<=5$ Jahre und $>5$ Jahre Behandlungsdauer). Auch die Angaben der Patienten zum Wohnort wurden in zwei Kategorien geteilt: „Dorf“ oder „Kleinstadt“ ( $n=76$ ) versus „größere Stadt“ oder „Großstadt“ ( $n=231$ ).

\subsection{Arztfragebogen}

Die Hausärzte wurden neben praxisstatistischen Daten zu folgenden Themen befragt (siehe Anhang):

- Häufigkeit von Sexualstörungen bei männlichen Patienten

- Ansprechen von Sexualstörungen durch den Hausarzt

- Möglichkeiten und Grenzen der Behandlung sexueller Störungen in der Hausarztpraxis

- Gründe für ein Nicht-Ansprechen möglicher sexueller Schwierigkeiten

- Bedeutung sexualmedizinischer Kenntnisse und von Sexualberatung

- Stellenwert von Sildenafil (Viagra ${ }^{\circledR}$ )

- Interesse an Fortbildungsveranstaltungen. 
Auch im Arztfragebogen wurden Zahlenwerte („Noten“) gegebenenfalls kategorial aufgeteilt:

- $\quad$ unwichtig bzw. sehr gering $(=1-3)$

- $\quad$ wichtig bzw. durchschnittlich (=4 - 7)

- $\quad$ sehr wichtig bzw. sehr gut $\quad(=8-10)$.

\subsection{Pilotstudie}

Der Fragebogen wurde in einer Allgemeinarztpraxis im Raum Göttingen pilotiert. An einem Praxistag wurden alle männlichen Patienten ab 18 Jahren um ihre Mitarbeit gebeten. 20 von 25 Männern erklärten sich bereit, den Fragebogen auszufüllen (Teilnehmerquote: $80 \%$ ); vorrangiger Grund der „Verweigerer“ war Zeitmangel.

Aufgrund der Pilotstudie wurde der Patientenfragebogen präzisiert. Beispielsweise wurde in der Frage nach der Zufriedenheit mit der Behandlung durch den Hausarzt (siehe Anhang 2) ausdrücklich darauf hingewiesen, daß hier nur die Betreuung bei sexuellen Problemen gemeint sei.

In ausführlichen Gesprächen mit drei niedergelassenen Fachärzten für Allgemeinmedizin aus der Region Göttingen wurde die „Brauchbarkeit“ des Arztfragebogens bewertet und anschließend modifiziert. U.a. wurde die Frage aufgenommen, ob die jeweiligen Partner in Gespräche über sexuelle Probleme miteinbezogen werden und ob dies auch gelingt (siehe Anhang 1).

\subsection{Auswahl der Arztpraxen}

Als Studienregionen wurden die Einzugsgebiete der Städte Hildesheim (Niedersachsen) und Heiligenstadt (Thüringen) gewählt, um Unterschiede zwischen städtisch und ländlich geprägten Praxen feststellen zu können (Einwohnerzahl Hildesheim: ca. 110.000; Heiligenstadt: ca. 15.500). 
Alle niedergelassenen Fachärzte für Allgemeinmedizin wurden mit Hilfe des Ärzteverzeichnisses schriftlich um ihre Mitarbeit gebeten. Einige Tage später wurde mit den Ärzten ein persönlicher Termin vereinbart, um den Patientenfragebogen sowie den geplanten Ablauf der Erhebung vorzustellen. Der Arztfragebogen sollte per beigelegtem Freiumschlag an die Abteilung Allgemeinmedizin zurückgesandt werden.

\subsection{Auswahl der Patienten und Ablauf der Patientenbefragung}

Vor Beginn der Datenerhebung wurden die Arzthelferinnen in den Hausarztpraxen über Sinn und Ziel der Befragung informiert. An einem zufällig gewählten Tag wurden die männlichen Patienten im Empfangsbereich der Praxis von der Doktorandin persönlich über die Studie informiert und anschließend gebeten, den Fragebogen auszufüllen. Die Information der Patienten im Empfangsbereich betonte die familienmedizinischen Aspekte des Fragebogens, um innen mögliche „Verlegenheiten“ in dem häufig stark frequentierten Anmeldebereich zu ersparen. Auch hatten die Hausärzte um Diskretion gebeten. Ausgeschlossen wurden Patienten unter 18 Jahren sowie Patienten mit unzureichenden Deutschkenntnissen, da sich der Fragebogen nicht dazu eignete, vorgelesen zu werden.

Die Patienten, die sich zur Mitarbeit bereit erklärten, wurden in ein separates Zimmer (meist das zweite Sprech- oder Wartezimmer) oder notfalls in eine abgetrennte Kabine (EKG-Kabine, Verbandsraum o.ä.) begleitet. Hier wurden sie genauer über den Zweck der Untersuchung (Beratung bei Sexualproblemen durch den Hausarzt) informiert. Die Patienten konnten anschließend den Fragebogen ungestört ausfüllen und ihn dann in die dazu vorgesehene „Box“ werfen. Die Patienten benötigten für das Ausfüllen des Bogens zwischen 5 Minuten und 2 Stunden (ein Extremfall), im Durchschnitt etwa 10 - 15 Minuten. 


\subsection{Auswertung}

Die Datenauswertung wurde mit dem Statistikprogramm SAS (1989) vorgenommen. Neben einfachen Häufigkeitsauszählungen wurden folgende Testverfahren (in Abhängigkeit von den statistischen Grundvoraussetzungen) zur Absicherung von Unterschieden bzw. Zusammenhängen durchgeführt:

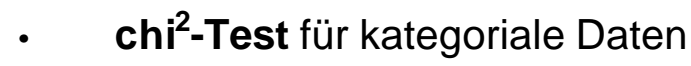

- $\quad$ t-Test für unabhängige Stichproben und paired-Test für abhängige Stichproben bei kontinuierlichen Meßwerten

- Korrelation nach Spearman bei Ordinaldaten.

Soweit die Antworten von Patienten mit den Aussagen von Ärzten in Beziehung gesetzt wurden, sind die Daten im Sinne eines nested designs ausgewertet worden. Als Analyseeinheit (unit of analysis) fungierte die jeweilige Arztpraxis. Die Aussagen aller Patienten einer Praxis wurden als eine Beobachtungseinheit (Mittelwert) behandelt. Diese Werte wurden z.B. mit den selbsteingeschätzten Kenntnissen der Hausärzte zur Sexualmedizin bzw. zur Einschätzung der Wichtigkeit dieser Thematik in Beziehung gesetzt (Korrelation nach Spearman). 


\section{Ergebnisse}

\subsection{Reaktionen auf die Untersuchung}

Die teilnehmenden Hausärzte standen unserer Untersuchung größtenteils sehr positiv gegenüber und betonten die Notwendigkeit der Thematisierung von Sexualproblemen in der Hausarztpraxis. Einige baten jedoch gleichzeitig um größtmögliche Diskretion („Aber machen Sie's diskret, sonst müssen wir das Ganze abbrechen. "). Insgesamt waren die Ärzte sehr interessiert an den Ergebnissen der Studie.

Eine anfängliche Skepsis der Arzthelferinnen resultierte aus der Vermutung, daß die Patienten eine Befragung ablehnen würden. Zugleich waren die meisten Helferinnen aber der Meinung, Sexualstörungen seien ein wichtiges und bisher vernachlässigtes Thema. Außerdem bedauerten einige, daß sich die Studie nur auf Männer bezog. Auch bei Frauen gäbe es einen Forschungsbedarf; zudem würden Frauen auf eine solche Studie aufgeschlossener reagieren als Männer.

Die meist gute, oft von Humor geprägte Zusammenarbeit mit den Arzthelferinnen (,....wir müßten öfter mal 'nen Männertag machen...") - stellte sich als wichtig und hilfreich für die Durchführung der Studie heraus (z.B. bei Zweifeln an der Volljährigkeit oder den Deutschkenntnissen eines Patienten). Daß die Helferinnen die Untersuchung unterstützten, erleichterte den Zugang zu den Patienten.

Die Patienten reagierten insgesamt sehr aufgeschlossen auf das Thema der Studie. Die Atmosphäre war überwiegend ernst und sachlich, teilweise deutete sich Scham seitens der Patienten an, ohne daß ein Zusammenhang zu bestimmten Altersgruppen hergestellt werden konnte. Manche Männer begannen, ihre Probleme in diesem Bereich zu erzählen. Nach dem Ausfüllen des Bogens berichteten Patienten, es hätte „Spaß gemacht", sich mit den Fragen zu beschäftigen, bzw. sei ihnen allein durch das Ausfüllen des Fragebogens „einiges klar geworden“. Einige Patienten kommentierten das Thema oder inhaltliche Aspekte des Fragebogens. Oft wurde angemerkt, daß über Sexualität bzw. Sexualprobleme nicht offen genug 
gesprochen werde und daß das Vertrauensverhältnis zum Hausarzt ein entscheidender Faktor für eine Betreuung und Behandlung bei diesen Problemen sei. Einige ältere Patienten merkten an, daß Sexualität in ihrem Alter kaum noch eine Rolle spiele, andere dagegen sahen sich und ihre Schwierigkeiten aufgrund des höheren Alters zu wenig ernstgenommen.

\section{2 Ärzte}

\subsubsection{Stichprobe}

Insgesamt wurden 40 Praxen von Fachärzten für Allgemeinmedizin in Hildesheim und neun Praxen in Heiligenstadt angeschrieben. Drei Ärzte aus Hildesheim wurden ausgeschlossen, da ihre Praxen aus Altersgründen nicht mehr existierten. In Hildesheim nahmen 17 Praxen (46\%) an der Untersuchung teil; in Heiligenstadt drei Praxen (33\%) (Tabelle 1). Acht Ärzte ließen bereits durch ihre Arzthelferinnen ausrichten, daß sie an der Studie nicht teilnehmen möchten, 15 teilten dies telephonisch selbst mit. Drei Ärzte lehnten eine Teilnahme nach persönlicher Vorstellung der Studie ab. Als Gründe wurden vor allem Zeitmangel, Überlastung der Praxis sowie Unzumutbarkeit für die Patienten genannt; einige Ärzte sorgten sich um ,psychisch labile" und „ältere" Patienten.

Die Teilnahmebereitschaft von Ärztinnen war sehr gering (Tabelle 1), obwohl z.B. in Heiligenstadt der Großteil der angeschriebenen Ärzte weiblich war (67\%). Auch in Hildesheim war die Teilnahmebereitschaft weiblicher Ärzte (25\%) deutlich geringer als die ihrer männlichen Kollegen (52\%). 
Tabelle 1: Teilnahmebereitschaft der Arztpraxen

\begin{tabular}{lccc}
\hline & Ärzte & Ärztinnen & Gesamt \\
& $\mathbf{n}$ & $\mathbf{n}$ & $\mathbf{n}$ \\
\hline Hildesheim & 31 & 9 & 40 \\
Angeschrieben & 2 & 1 & 3 \\
Ausschluß & 14 & 6 & 20 \\
Ablehnungen & $15(52 \%)$ & $2(25 \%)$ & $17(46 \%)$ \\
Zusagen & & & \\
Heiligenstadt & 3 & 6 & 9 \\
Angeschrieben & - & - & - \\
Ausschluß & 1 & 5 & 6 \\
Ablehnungen & $2(67 \%)$ & $1(17 \%)$ & $3(33 \%)$ \\
Zusagen & & & \\
Gesamtstichprobe & 34 & 15 & 49 \\
Angeschrieben & 2 & 1 & 3 \\
Ausschluß & 15 & 11 & 26 \\
Ablehnungen & $17(53 \%)$ & $3(21 \%)$ & $20(43 \%)$ \\
Zusagen & & & \\
\hline
\end{tabular}

Der Altersdurchschnitt aller teilnehmenden Ärzte lag bei 49 Jahren (min: 36 Jahre, max: 61 Jahre). Die Ärzte waren im Durchschnitt seit 12 Jahren niedergelassen (min: 1 Jahr, max: 22 Jahre). Fast die Hälfte der Ärzte (44\%) gab eine Scheinzahl von 500-999 je Quartal an (siehe Grundauszählung im Anhang). Zehn Allgemeinärzte besaßen Zusatzbezeichnungen, davon zwei die Zusatzbezeichnung „Psychotherapie“.

\subsubsection{Arztfragebogen}

Aus 16 der 17 teilnehmenden Hildesheimer Praxen sandten die Ärzte den ArztFragebogen ausgefültt zurück (94\%); in Heiligenstadt waren es alle drei (100\%). Die nachfolgenden Zahlen beziehen sich auf diese 19 Praxen. 


\subsubsection{Häufigkeit von Sexualstörungen in der Hausarztpraxis}

Nach eigenen Angaben wurden die befragten Ärzte von durchschnittlich sieben Patienten im Quartal primär wegen sexueller Störungen aufgesucht. Bei weiteren sechs Patienten im Quartal ergaben sich Hinweise auf sexuelle Schwierigkeiten. Bei durchschnittlich zehn Patienten im Quartal sahen die Hausärzte Sexualprobleme als den eigentlichen Grund für die Konsultation, während diese Patienten einen anderen Konsultationsgrund (z.B. Blasenentzündung) vorschieben würden.

\subsubsection{Ansprechen von Sexualproblemen durch den Hausarzt}

Jeder zweite Arzt (53\%) gab an, seine Patienten manchmal auf möglicherweise bestehende Sexualstörungen anzusprechen; ein Drittel der Ärzte (37\%) tat dies dagegen nur selten (Abbildung 1).

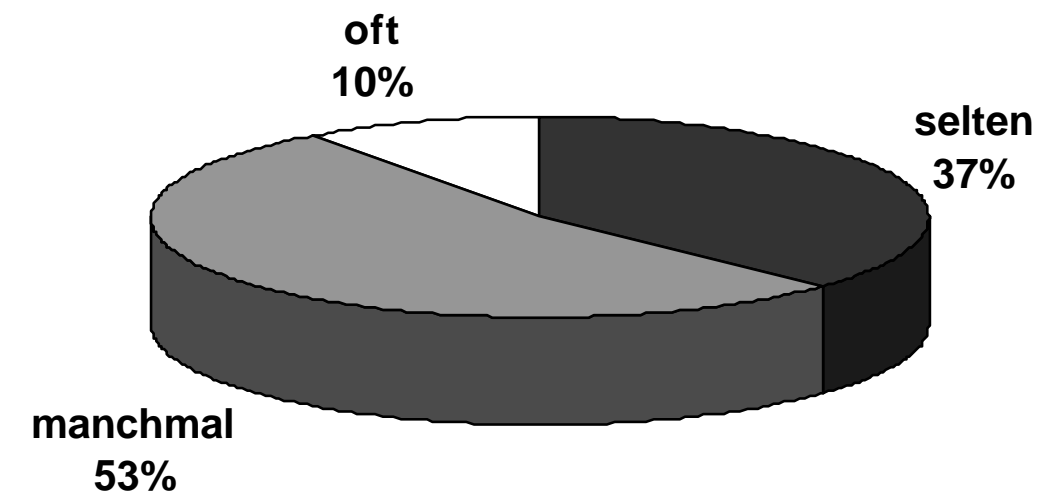

Abbildung 1: Wie häufig initiieren Hausärzte ein Gespräch bei möglichen Sexualstörungen? $(n=19)$

In den Tabellen 2 und 3 sind die Anlässe bzw Krankheiten aufgeführt, bei denen Ärzte ihre Patienten auf mögliche Sexualstörungen ansprechen. Die Ärzte wurden 
gefragt, bei welchen Anlässen bzw. Krankheiten sie ihre Patienten auf mögliche Sexualstörungen ansprechen, nicht jedoch, ob sie dies bei den aufgelisteten Krankheitsbildern grundsätzlich tun.

Tabelle 2: Anlässe für Gespräche über mögliche Sexualprobleme ( $n=19$; Mehrfachnennungen)

\section{Anlaß}

in $\%$

Bei psychosomatischen Beschwerden

84

Im Zusammenhang mit Fragen zur Familienplanung

53

Bei Fragen zu HIV

47

Grundsätzlich bei jeder Gesundheitsuntersuchung

16

Tabelle 3: Gespräche über Sexualität bei Vorliegen bestimmter Krankheiten ( $n=19 ;$ Mehrfachnennungen)

Krankheiten

Diabetes mellitus

Hypertonie

Prostataadenom

hormonelle Störungen

Arteriosklerose

Schlafstörungen

Multiple Sklerose

Migräne in $\%$ 79

63

58

42

37

26

11

5 
Der überwiegende Teil der Ärzte gab an, ihre Patienten bei einer Medikation mit Antihypertonika (84 \%) und Psychopharmaka (63\%) auf Sexualstörungen anzusprechen. Bei einer Therapie mit Diuretika lag der Anteil bei $16 \%$.

\subsubsection{Gründe für ein Nicht-Ansprechen sexueller Probleme}

Jeder zweite Arzt (53 \%) nannte Zeitmangel als Grund, Sexualprobleme im Praxisalltag nicht anzusprechen. Auch „unzureichende Kenntnisse“ auf diesem Gebiet oder „nicht daran zu denken“ waren häufige Gründe. Schamgefühle nannten $23 \%$ der Ärzte, (90\% der Ärzte vermuteten dies als Grund auf Seiten der Patienten). In ähnlicher Weise sahen $74 \%$ der Hausärzte Formulierungsschwierigkeiten seitens der Patienten als Hindernisgrund, aber nur $26 \%$ der Hausärzte sahen Formulierungsschwierigkeiten bei sich selbst. Immerhin jeder fünfte Arzt wertete mangelndes Vertrauen und etwa jeder siebte die Angst, den Arzt zu „enttäuschen“, als Grund für die Patienten, ihren Hausarzt bei möglichen sexuellen Problemen nicht zu Rate zu ziehen (Tabelle 4).

Tabelle 4: Gründe für ein Nicht-Ansprechen möglicher sexueller Probleme aus Sicht des Arztes ( $n=19$; Mehrfachnennungen)

Gründe (nach Arzteinschätzung)

in $\%$

\section{Arztbezogene Faktoren}

Zeitmangel

Unzureichende Kenntnisse

Nicht daran gedacht

Schamgefühle 32

Formulierungsschwierigkeiten 26

\section{Patientenbezogene Faktoren}

Schamgefühle 90

Formulierungsschwierigkeiten

Mangelndes Vertrauen zum Arzt

Angst, den Arzt zu „enttäuschen“ 


\subsubsection{Bedeutung sexualmedizinischer Kenntnisse und von Sexualberatung}

Die Ärzte bewerteten die Bedeutung sexualmedizinischer Kenntnisse für die hausärztliche Tätigkeit mit 7 und die Wichtigkeit von Sexualberatung mit 6 (1 = unwichtig; 10 = sehr wichtig). Nach der Beurteilung der eigenen sexualmedizinischen Kenntnisse gefragt, gaben sich die Hausärzte im Durchschnitt 6 Punkte. Die Ärzte beurteilten ihre eigenen sexualmedizinischen Kenntnisse im Durchschnitt um 1,15 Punkte geringer als die Bedeutung dieser Kenntnisse in der Hausarztpraxis. Tabellen 5 und 6 illustrieren diese Einschätzungen auf Basis einer „Drittelung“ der Zehnerskalen (s. S. 20)

Tabelle 5: Bedeutung sexualmedizinischer Kenntnisse und von Sexualberatung in der Hausarztpraxis aus Sicht des Arztes $(n=19)$

\begin{tabular}{lcc}
\hline Bedeutung ${ }^{1)}$ & $\begin{array}{c}\text { Sexualmedizinische } \\
\text { Kenntnisse } \\
\text { in } \%\end{array}$ & $\begin{array}{c}\text { Sexualberatung } \\
\text { in \% }\end{array}$ \\
\hline Unwichtig & 0 & 17 \\
Wichtig & 53 & 56 \\
Sehr wichtig & 47 & 28 \\
${ }^{1)}$ unwichtig = 1-3; wichtig = 4-6; sehr wichtig = 7-10; s. S. 20 &
\end{tabular}

Tabelle 6: Beurteilung der eigenen sexualmedizinischen Kenntnisse

Beurteilung 1)

Sehr gering

Mittelmäßig

Sehrgut

\section{Beurteilung eigener Kenntnisse} in $\%$

\section{5}

84

11

\footnotetext{
1) sehr gering = 1-3; durchschnittlich = 4-6; sehr gut = 7-10; s. S. 20
} 


\subsubsection{Behandlung von Sexualproblemen in der Hausarztpraxis}

Etwa jeder zweite der befragten Hausärzte gab an, Erektionsstörungen bzw. Libidostörungen ( $61 \%$ bzw. 50 \%) in seiner Praxis behandeln zu können. Ejaculatio praecox wurde von $33 \%$ der Ärzte als in der Praxis behandelbar angesehen und der verzögerte Samenerguß von $17 \%$. Bei Vorliegen einer Sexualstörung änderten die Ärzte vor allem die Medikation (79\%) oder überwiesen an den Urologen (74\%).Immerhin die Hälfte zog eine Behandlung mit Sildenafil in Betracht (Tabelle 7).

Tabelle 7: Behandlung sexueller Störungen in der Hausarztpraxis

\begin{tabular}{ll}
\hline Behandlung durch & in \% \\
\hline Medikationsänderung & 79 \\
Überweisung an Urologen & 74 \\
Information und Gespräche & 58 \\
Sildenafil (Viagra ${ }^{\circledR}$ & 53 \\
Hormonsubstitution & 47 \\
Überweisung an Psychotherapeuten & 32 \\
Schwellkörperinjektion & 11 \\
Vakuumapparate & - \\
\hline
\end{tabular}

Nach eigenen Angaben versuchte etwas über die Hälfte der Hausärzte manchmal oder häufiger die Partner der Patienten in die Behandlung sexueller Störungen mit einzubeziehen; nach Selbsteinschätzung der Mehrheit der Ärzte gelingt dies aber nur selten oder nie (Abbildung 2). 


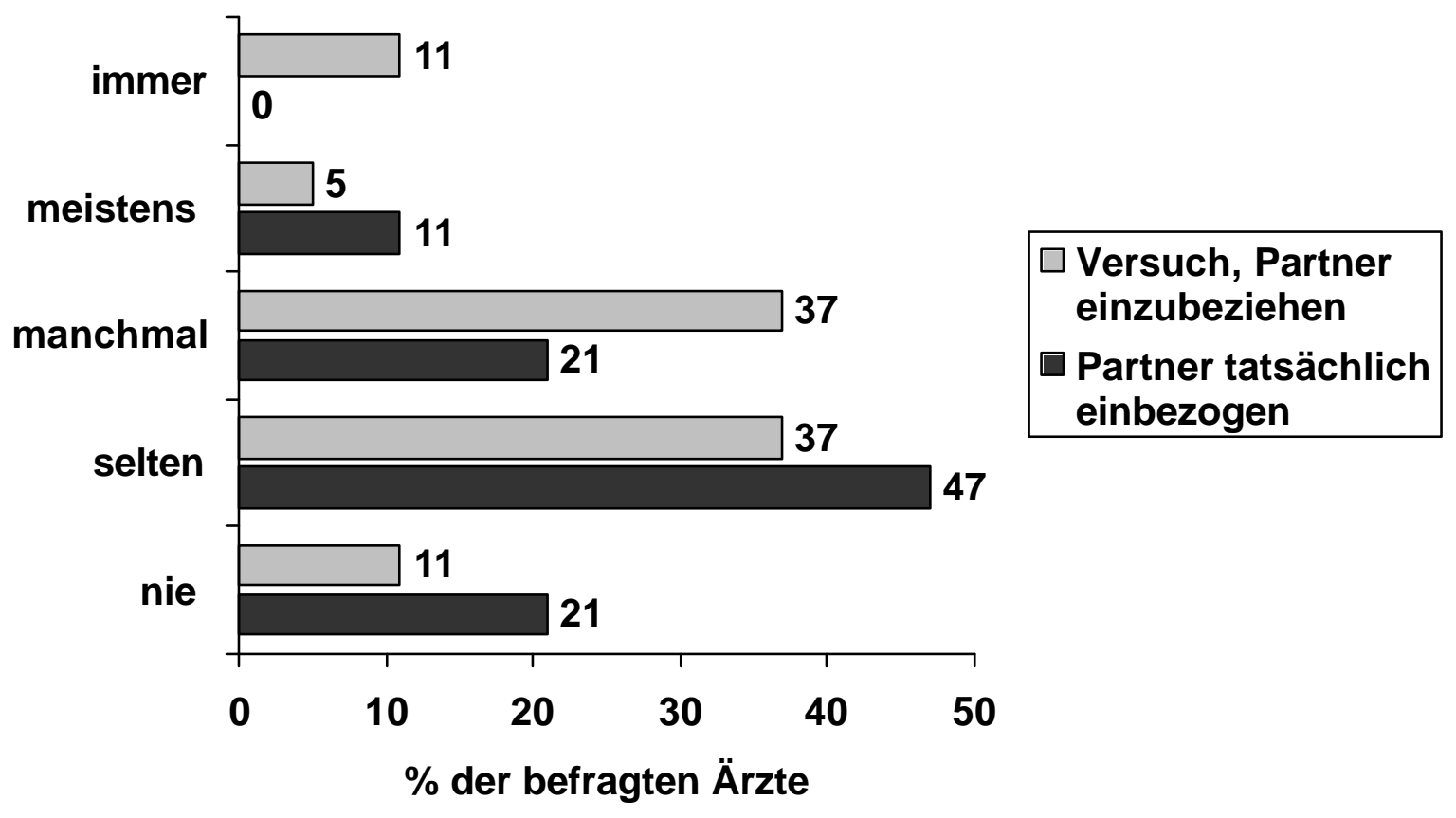

Abbildung 2: Wie häufig werden Partner in die Behandlung sexueller Funktionsstörungen einbezogen?

\subsubsection{Stellenwert von Sildenafil $\left(\right.$ Viagra $\left.^{\circledR}\right)$}

Weit über ein Drittel der Hausärzte (42\%) war der Meinung, die Behandlung mit Sildenafil $\left(\right.$ Viagra $^{\circledR}$ ) lenke bei Sexualstörungen von den eigentlichen psychischen Problemen des Patienten ab. Ein Viertel der Ärzte (26\%) sah in Viagra ${ }^{\circledR}$ ein gutes Mittel, Potenzprobleme zu lösen, bevor es zu gravierenden psychischen Problemen komme. Jeder fünfte Arzt hielt die Diskussion um Nebenwirkungen von Viagra ${ }^{\circledR}$ in Anbetracht der starken psychischen Belastungen von Patienten mit Erektionsstörungen für unangemessen. $16 \%$ der Ärzte würden dagegen aufgrund der unerwünschten Nebenwirkungen Viagra ${ }^{\circledR}$ nur in schwersten Fällen erektiler Dysfunktion verordnen. Insgesamt $79 \%$ der Ärzte bejahten die generelle Frage, ob sie Viagra ${ }^{\circledR}$ verschreiben würden.

Fast jeder zweite Hausarzt (47\%) gab an, daß sich im Zusammenhang mit der öffentlichen Diskussion um Viagra ${ }^{\circledR}$ häufiger Beratungsanlässe über Potenzprobleme bzw. Sexualfragen ergeben hätten. 


\subsubsection{Fortbildungswünsche der Hausärzte}

$78 \%$ der befragten Ärzte würden sich Fortbildungsveranstaltungen zum Thema Diagnostik und Therapie von Sexualstörungen wünschen - vor allem zu den folgenden Störungen:

- Erektile Dysfunktion

- Ejaculatio praecox

- Ejaculatio retardata

- Libidostörungen.

\subsection{Patienten}

\subsubsection{Stichprobe}

419 Patienten wurden in den Studienpraxen um Mitarbeit gebeten; letztlich füllten 307 Patienten den Fragebogen aus (Tabelle 8). 55 Patienten wurden wegen unzureichender Deutschkenntnisse oder Sehbehinderung von der Befragung ausgeschlossen. 57 Patienten verweigerten die Teilnahme - vorwiegend aus Zeitmangel, weil sie sich zu krank fühlten oder „keine Lust" hatten. Die meisten Verweigerungen $(n=40)$ wurden bereits bei der Kontaktaufnahme im Empfangsbereich der Praxis ausgesprochen. Da zu diesem Zeitpunkt über den genauen Inhalt des Fragebogens noch nichts mitgeteilt worden war, kann das Thema "Sexualität" kaum der Grund für die Ablehnung gewesen sein. Fünf Patienten verweigerten ihre Teilnahme, nachdem sie den Fragebogen erhalten hatten: wegen hohen Alters (,...damit hab' ich nichts mehr zu tun...") oder aus Zeitmangel. 43 Patienten konnten nicht befragt werden, da das Konsultationsgespräch unmittelbar bevorstand und der Praxisablauf nicht verzögert werden sollte. 
Tabelle 8: Teilnahmebereitschaft der Patienten

\begin{tabular}{lccc}
\hline & Hildesheim & Heiligenstadt & Gesamt \\
\hline Angesprochen; n & 388 & 31 & 419 \\
Ausschluß; n (\%) & $52(13)$ & $3(10)$ & $55(13)$ \\
Ablehnung; n (\%) & $53(16)$ & $4(14)$ & $57(16)$ \\
Teilnahme ; n (\%) & $283(84)$ & $24(86)$ & $307(84)$ \\
\hline
\end{tabular}

Im Durchschnitt füllten 15 Patienten pro Praxis (min: 3 Patienten, max: 27 Patienten) den Fragebogen aus. Entsprechend der unterschiedlichen Größe der Teilstichproben (s. Kap. 4) nahmen 283 Patienten in Hildesheim und 24 in Heiligenstadt teil. Im Durchschnitt waren die befragten Patienten 45 Jahre alt. Tabelle 9 zeigt das Alter der teilnehmenden Patienten im Vergleich mit dem Alter der männlichen Patienten von Allgemeinärzten des ADT-Panels des Zentralinstituts für Kassenärztliche Versorgung (Kerek-Bodden et al. 2000). Diese Studie basiert auf anonymisierten Abrechnungsdaten.

Tabelle 9: Alter der teilnehmenden Patienten

\begin{tabular}{|c|c|c|}
\hline Jahre & $\begin{array}{c}\text { eigene Studie in \% } \\
n=285^{\star}\end{array}$ & $\begin{array}{c}\text { ADT-Panel in \%** } \\
\text { n=24924 }\end{array}$ \\
\hline $20-29$ & 14 & 14 \\
\hline $30-39$ & 22 & 20 \\
\hline $40-49$ & 22 & 15 \\
\hline $50-59$ & 19 & 17 \\
\hline $60-69$ & 16 & 19 \\
\hline $70-79$ & 6 & 11 \\
\hline $80-84$ & 1 & 4 \\
\hline
\end{tabular}


Überwiegend hatten die Patienten einen Hauptschulabschluß (33\%) oder Realschulabschluß (31\%).

Tabelle 10 gibt einen Überblick über Familienstand, Wohnort und Beruf der befragten Patienten. Die relativ häufige Angabe von „Kleinstadt" und „Dorr" spiegelt die eingemeindeten Stadtteile von Hildesheim, deren Einwohner sich als Dorfbewohner einstuften.

Tabelle 10: Familienstand, Wohnort und Beruf der befragten Patienten

Soziodemographische Daten

n (\%)

\section{Familienstand}

Verheiratet $195(64)$

Feste Partnerschaft $65(21)$

Alleinstehend $37(12)$

Geschieden 5 ( 2)

Verwitwet

Wohnort (Einwohnerzahl)

Dorf $(<5.000)$ 28 ( 9)

Kleinstadt (5.000-20.000) 48 (16)

Größere Stadt (20.000-100.000) 90 (30)

Großstadt (>100.000) $133(45)$

\section{Beruf}

In Ausbildung/Studium

$31(10)$

Arbeiter

$60(20)$

Angestellter

$89(30)$

Beamter

$13(5)$

Arbeitsloser

$22(7)$

Rentner

$70(24)$

Selbständiger

8 ( 3$)$

ohne nähere Bezeichnung

4( 1$)$ 
69 \% der Männer hatten Kinder. Einen bis dahin unerfüllten Kinderwunsch gaben $6 \%$ an, wobei sich zwei Drittel seit 1-2 Jahren ein Kind wünschten, die übrigen seit 10 Jahren oder länger. Ein Drittel der Patienten (34\%) litt unter einer oder mehreren chronischen Krankheiten (Tabelle 11). Hier wurden Herz-Kreislauf-Krankheiten und Stoffwechselkrankheiten (v.a. Diabetes mellitus) am häufigsten genannt.

Tabelle 11: Chronische Krankheiten ( $n=291$; Mehrfachnennungen)

\begin{tabular}{lc}
\hline Krankheit & in \% \\
\hline Herz-Kreislauf-Erkrankungen & 11 \\
Stoffwechselkrankheiten & 10 \\
Bewegungsapparat & 4 \\
Atemwegserkrankungen & 4 \\
Neurologische Erkrankungen & 2 \\
Allergien & 2 \\
\hline
\end{tabular}

\subsubsection{Konsultationsanlaß und Beziehung zum Hausarzt}

Überwiegend hatten die befragten Patienten ihren Hausarzt am Tag der Erhebung aufgrund akuter oder chronischer Erkrankungen aufgesucht (Tabelle 12). $90 \%$ der Männer waren seit mehr als einem Jahr bei ihrem Hausarzt in Behandlung, zumeist schon über 5 Jahre (max: 41 Jahre). $83 \%$ der Patienten meinten, ihr Hausarzt sei über ihre persönlichen Lebensumstände, wie Beruf, Wohnsituation und familiäre Verhältnisse, informiert. Jeder Zweite habe inm von sich aus hierüber berichtet. In $22 \%$ der Fälle hätte der Hausarzt selber danach gefragt. Nur $5 \%$ der Patienten fanden, daß ihre persönlichen Lebensumstände den Hausarzt nichts angingen (Tabelle 13). 
Tabelle 12: Konsultationsanlaß ( $n=299$; Mehrfachnennungen)

\begin{tabular}{lc}
\hline Konsultationsanlaß & in \% \\
\hline Akute Erkrankung & 45 \\
Chronische Krankheit & 40 \\
Vorsorgeuntersuchung & 14 \\
Beratung & 8 \\
Sonstiges & 18 \\
\hline
\end{tabular}

Tabelle 13: Kenntnis des Hausarztes über die persönlichen Lebensumstände ( $n=298$; Mehrfachnennungen)

Kenntnis des Hausarztes $\quad$ in \%

... ich habe inm von mir aus darüber berichtet $\quad 51$

... der Hausarzt hat danach gefragt 22

... das hat sich so ergeben $\quad 30$

... das geht inn nichts an $\quad 5$

... ich kann darüber mit inm nicht sprechen 2

... ich denke, daß er darüber nicht sprechen möchte 3

\subsubsection{Rolle des Hausarztes aus Patientensicht}

$79 \%$ der Patienten meinten, bei Bedarf problemlos mit ihrem Hausarzt über Sexualprobleme sprechen zu können; für $84 \%$ wäre dies auch wichtig. Knapp die Hälfte der Patienten (45\%) wünschte, ihr Hausarzt möge sie von sich aus auf mögliche Schwierigkeiten in Sexualität und Partnerschaft ansprechen. Die Bereitschaft zu solchen Gesprächen könne der Hausarzt vor allem durch direktes Ansprechen bzw. Nachfragen während einer Behandlung signalisieren, meinten $64 \%$. Auch das Auslegen von Informationsbroschüren und Büchern zum Thema Sexualprobleme sei eine gute Möglichkeit, Gesprächsbereitschaft zu zeigen (51\%). 
Für mehr als die Hälfte der Patienten (56\%) spielte das Geschlecht des Hausarztes für Gespräche über sexuelle und partnerschaftliche Probleme keine Rolle. Dies gilt auch, wenn man die Praxen mit weiblichem bzw. männlichem Hausarzt getrennt betrachtet (Abbildung 3).

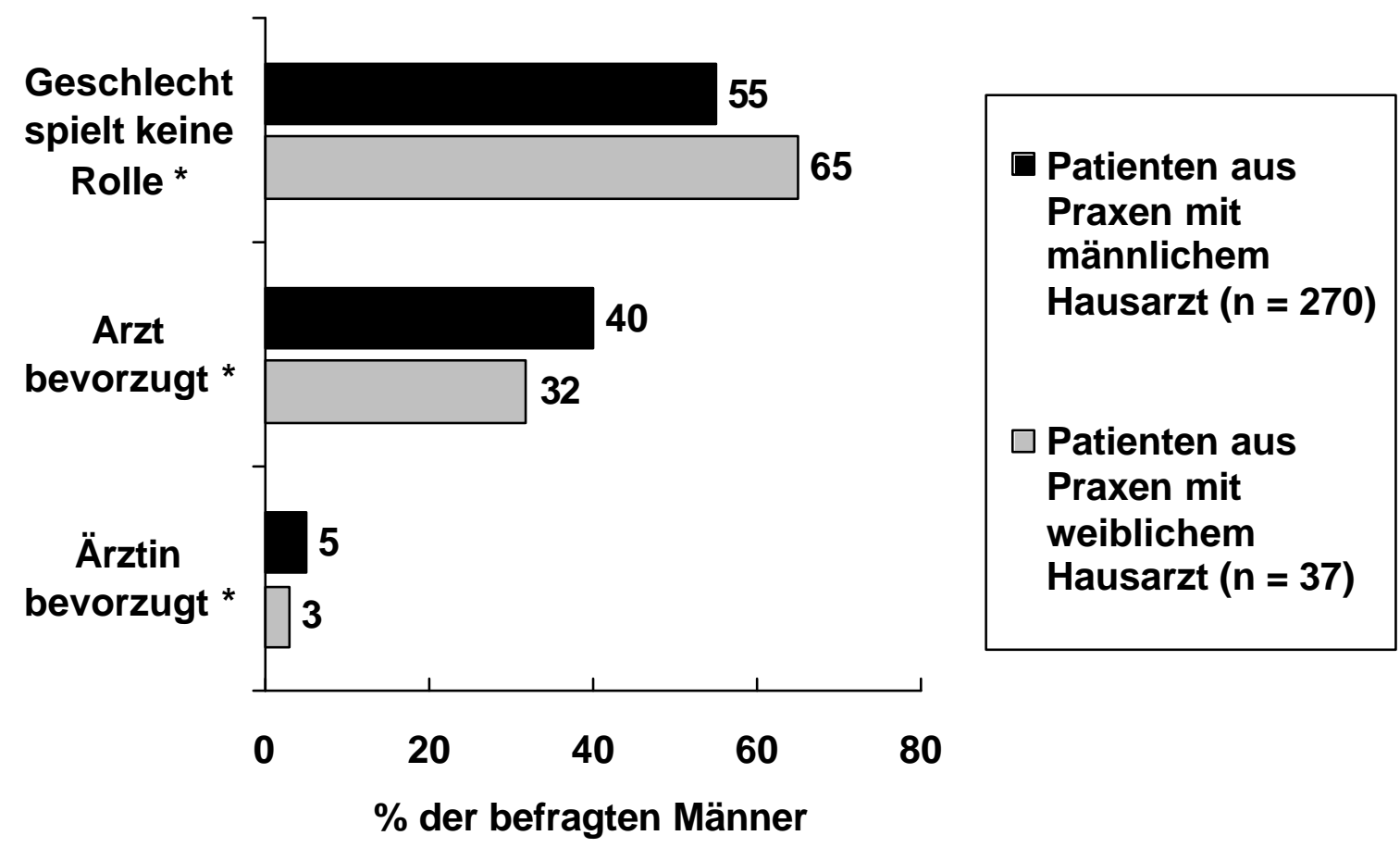

Abbildung 3: Die Bedeutung des Geschlechts des Hausarztes für Gespräche über Sexualität $(n=291) ;{ }^{*}$ nicht signifikant

\subsubsection{Häufigkeit, Ausprägung und Ursachen sexueller Probleme}

Fast jeder zweite Patient (46\%) war nach eigenen Angaben sehr zufrieden mit dem eigenen Sexualleben (Werte von 8-10 auf einer 10-Punkte Lickert-Skala); 17 \% äuBerten sich sehr unzufrieden mit Werten von 1-3 (Abbildung 4). 


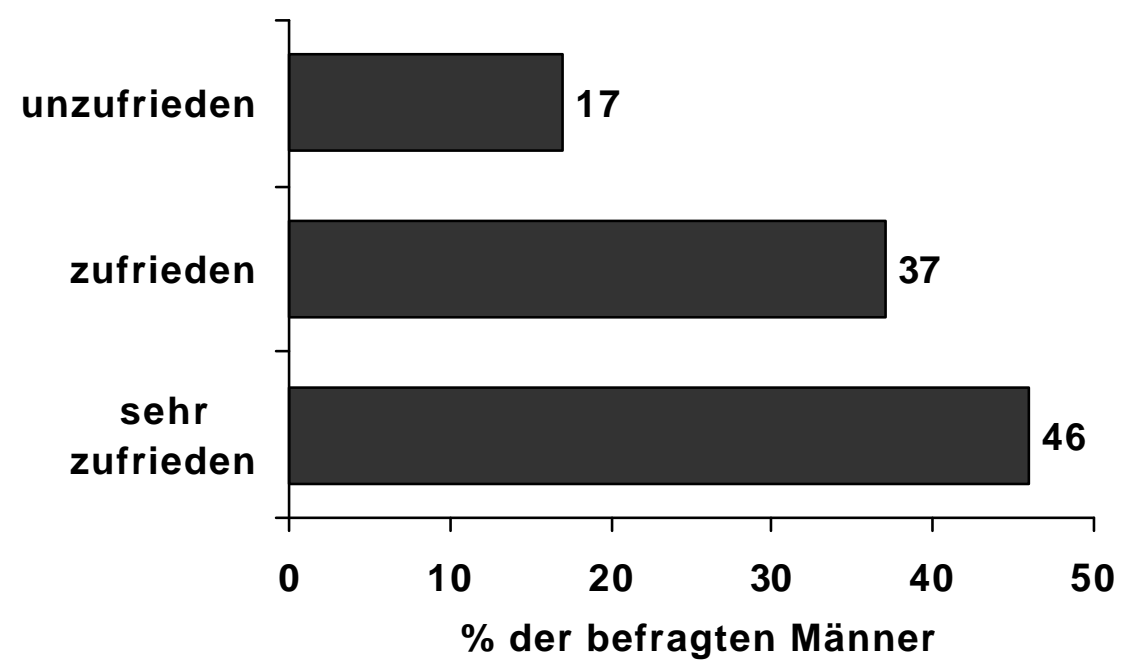

Abbildung 4: Zufriedenheit mit dem Sexualleben ${ }^{1)}$

1) unzufrieden = 1-3; zufrieden $=4-7$; sehr zufrieden $=8-10$; s. S. 19

Befragt nach konkreten Sexualproblemen gaben $7 \%$ der Patienten an, nie unter einer der aufgeführten Störungen zu leiden. Die am häufigsten genannten Probleme waren Lustlosigkeit und Ejaculatio praecox (Tabelle 14). 37 Männer (12\%) beantworteten diese Frage nicht.

Abbildung 5 zeigt die Gesamt-Häufigkeit von Sexualproblemen. Hierbei wurde von dem „höchstgenannten“ Wert ausgegangen: Gab ein Mann beispielsweise an, selten unter Erektionsstörungen und häufig unter Ejaculatio praecox zu leiden, wurde er nur der Kategorie häufig zugeordnet. 73 \% der Männer litten danach selten oder manchmal unter Sexualproblemen.

Abbildung 6 zeigt die Anzahl von Sexualschwierigkeiten der befragten Männer unabhängig von der Häufigkeit. 49 \% der Männer nannten mindestens 3 Probleme; z.B. litten $22 \%$ unter Lustlosigkeit, Versagensängsten und vorzeitigem Samenerguß. 
Tabelle 14: Häufigkeit und Ausprägung sexueller Probleme (Mehrfachnennungen)

\begin{tabular}{|c|c|c|c|c|c|}
\hline Patient leidet unter & $\begin{array}{l}\text { nie } \\
\%\end{array}$ & $\begin{array}{l}\text { selten } \\
\%\end{array}$ & $\underset{\%}{\operatorname{manchmal}}$ & $\begin{array}{c}\text { häufig } \\
\%\end{array}$ & $\underset{\%}{\operatorname{immer}}$ \\
\hline $\begin{array}{l}\text { Lustlosigkeit } \\
(\mathrm{n}=243)\end{array}$ & 27 & 34 & 34 & 3 & 2 \\
\hline $\begin{array}{l}\text { Ejaculatio praecox } \\
(n=218)\end{array}$ & 34 & 26 & 29 & 10 & 1 \\
\hline $\begin{array}{l}\text { Versagensängsten } \\
(n=218)\end{array}$ & 50 & 32 & 13 & 5 & 0 \\
\hline $\begin{array}{l}\text { Ejaculatio retardata } \\
(\mathrm{n}=199)\end{array}$ & 51 & 33 & 12 & 3 & 1 \\
\hline $\begin{array}{l}\text { Erektionsstörungen } \\
(\mathrm{n}=221)\end{array}$ & 62 & 22 & 10 & 4 & 2 \\
\hline $\begin{array}{l}\text { Ausbleibendem } \\
\text { Samenerguß } \\
(n=203)\end{array}$ & 75 & 16 & 7 & 1 & 1 \\
\hline \multirow[t]{2}{*}{$\begin{array}{l}\text { Schmerzen beim } \\
\text { Geschlechtsverkehr } \\
(n=202)\end{array}$} & 83 & 11 & 5 & 1 & 0 \\
\hline & $\begin{array}{l}\text { ja } \\
\%\end{array}$ & & $\begin{array}{c}\text { etwas } \\
\%\end{array}$ & & 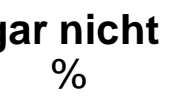 \\
\hline $\begin{array}{l}\text { Zu kleinem Penis } \\
(\mathrm{n}=188)\end{array}$ & 2 & & 14 & & 84 \\
\hline $\begin{array}{l}\text { Fehlbildungen des Penis } \\
(\mathrm{n}=192)\end{array}$ & 0 & & 6 & & 94 \\
\hline
\end{tabular}




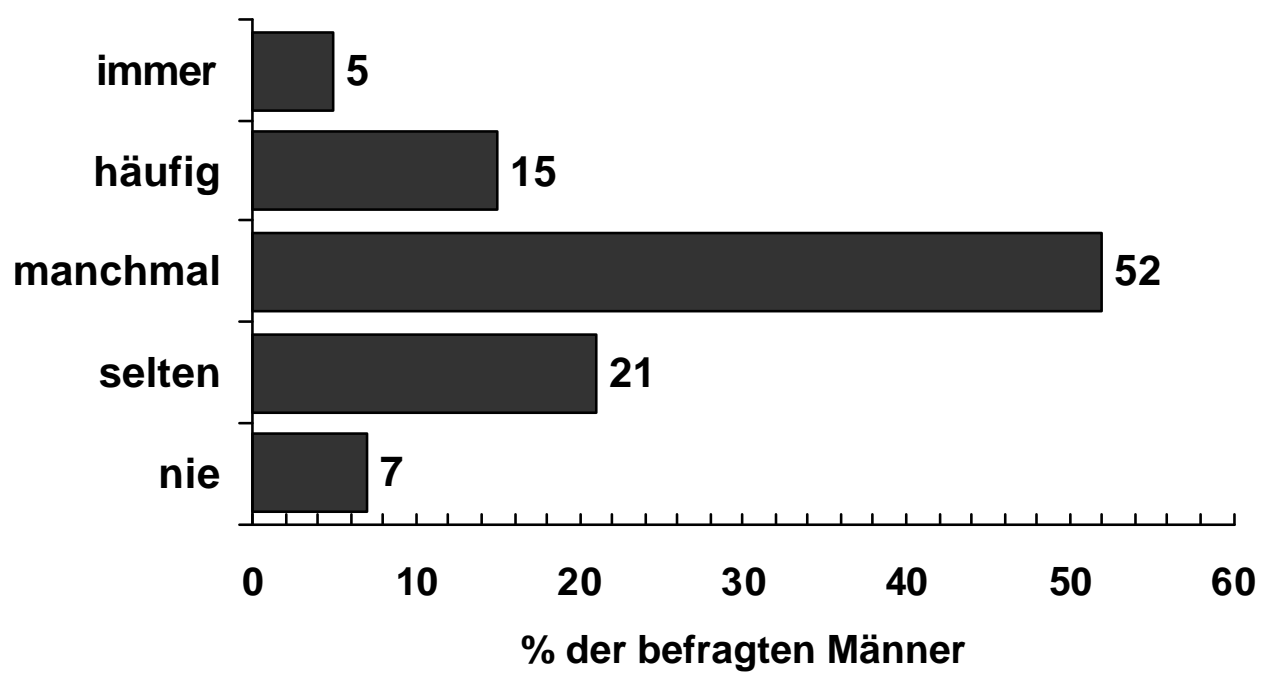

Abbildung 5: Häufigkeit von Sexualproblemen

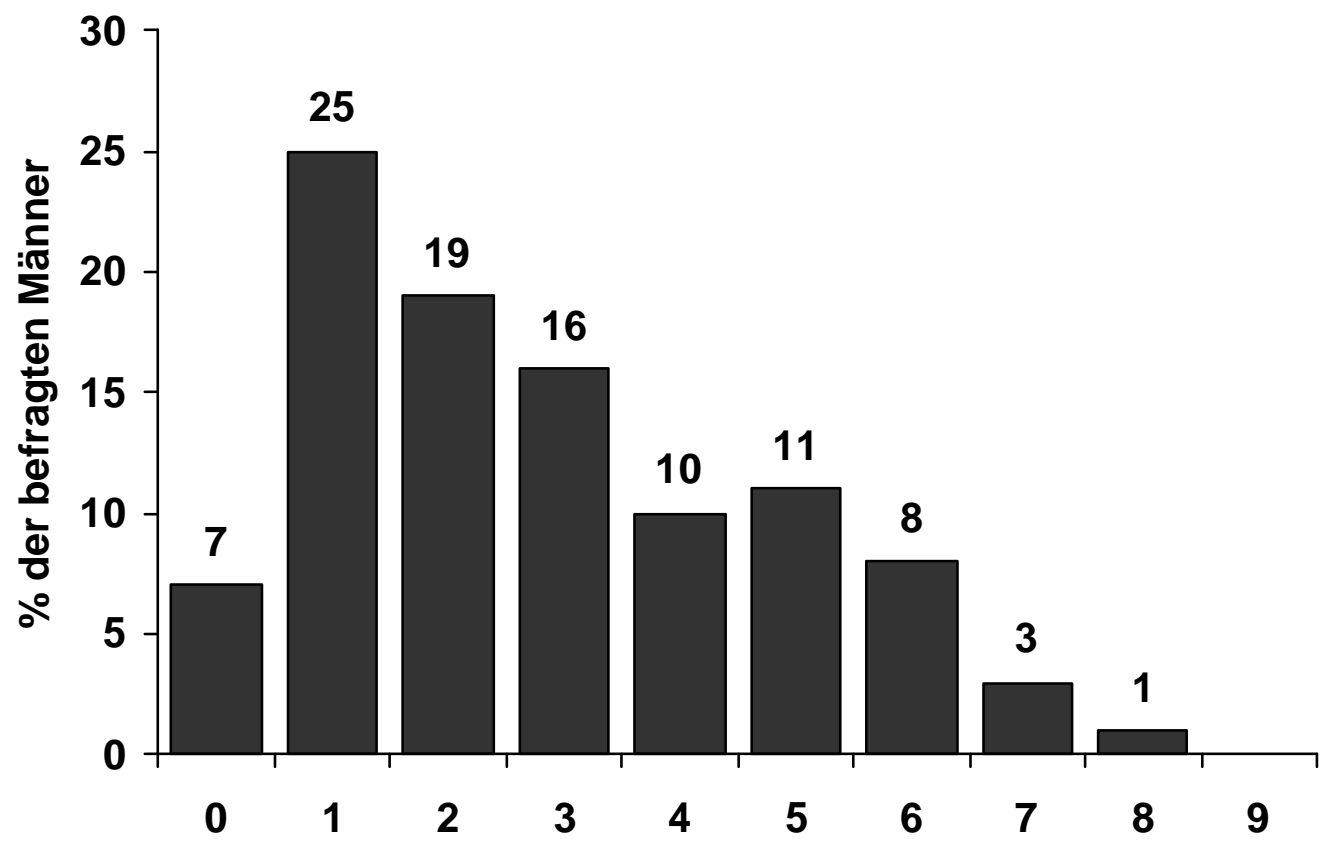

Anzahl der Sexualstörungen

Abbildung 6: Anzahl von Sexualproblemen 
$42 \%$ der Männer konnten zur Dauer ihrer Schwierigkeiten keine Angaben machen, $30 \%$ gaben einen Zeitraum von 1 bis 5 Jahren an; $19 \%$ litten seit mehr als sechs Jahren unter Sexualstörungen, größtenteils „schon immer“.

Fast die Hälfte der Patienten (45 \%) konnte keinen Grund für die sexuellen Schwierigkeiten angeben. 34 \% der Männer nannten eher seelische, 21 \% eher körperliche Gründe. Befragt nach den konkreten Ursachen für ihre Sexualprobleme, gab mehr als die Hälfte der befragten Männer beruflichen Streß an (Tabelle 15). Auch eigener Erwartungsdruck und Schwierigkeiten in der Partnerschaft spielten eine Rolle. Tabelle 16 zeigt die Auswirkungen der Sexualprobleme auf die Patienten, zu denen neben Schlafstörungen z.B. auch depressive Symptome zählten.

Tabelle 15: Ursachen der Sexualprobleme ( $n=201$; Mehrfachnennungen)

\begin{tabular}{lc}
\hline Ursachen & in \% \\
\hline Beruflicher Streß & 53 \\
Eigener Erwartungsdruck & 28 \\
Schwierigkeiten in der Partnerschaft & 19 \\
Eigene Krankheit & 13 \\
Medikamente & 9 \\
Hemmungen aufgrund strenger Sexualerziehung & 8 \\
Erwartungsdruck von anderen & 8 \\
Unerfülter Kinderwunsch & 3 \\
\hline
\end{tabular}


Tabelle 16: Auswirkungen der Sexualstörungen ( $n=257$; Mehrfachnennungen)

\begin{tabular}{lc}
\hline Auswirkungen & in \% \\
\hline Sexuelle Unzufriedenheit & 18 \\
Schlafstörungen & 14 \\
Schwierigkeiten in der Partne rschaft & 14 \\
Niedergeschlagenheit & 13 \\
Minderwertigkeitsgefühle & 7 \\
Depressionen & 5 \\
\hline
\end{tabular}

\subsubsection{Betreuung bei Sexualproblemen durch den Hausarzt}

Bei sexuellen Fragen und Problemen würden sich die meisten Befragten an ihre Partner wenden, ein Drittel an den Hausarzt (Tabelle 17).

Tabelle 17: Mögliche Ansprechpartner bei Problemen im sexuellen Bereich ( $n=274$; Mehrfachnennungen)

\begin{tabular}{lc}
\hline Ansprechpartner & in \% \\
\hline Partner & 69 \\
Hausarzt & 37 \\
Freunde & 28 \\
Urologe & 12 \\
Pastor/Pfarrer & 2 \\
Beratungsstelle & 2 \\
Selbsthilfegruppe & 1 \\
Sonstige & 3 \\
\hline
\end{tabular}

$10 \%$ der befragten Patienten wußten niemanden, an den sie sich bei sexuellen Fragen und Problemen wenden könnten. 
Etwa jeder zehnte Patient hatte wegen sexueller Probleme oder deren Folgen auch tatsächlich seinen Hausarzt aufgesucht, 59 \% von ihnen besprachen mit ihm ihre Schwierigkeiten, $31 \%$ erhielten Medikamente und $25 \%$ wurden an Spezialisten überwiesen.

Die Zufriedenheit mit diesem Aspekt hausärztlicher Betreuung lag im Durchschnitt bei 6,3 auf einer Skala von 1 (sehr unzufrieden) bis 10 (sehr zufrieden). Bei Drittelung dieser Skala (s. S. 19), wurde deutlich, daß die Patienten überwiegend sehr zufrieden (50 \%) bis zufrieden (22 \%) waren. Immerhin $28 \%$, (9 Männer) äuBerten jedoch starke Unzufriedenheit.

\subsubsection{Wünsche an den Hausarzt}

Befragt nach ihren Wünschen an den Hausarzt im Umgang mit Sexualproblemen möchte jeder zweite Patient mit seinem Hausarzt bei Bedarf offen und direkt über sexuelle Fragen und Probleme sprechen können. Auch Informationen über mögliche Behandlungsmethoden und ein ausreichendes Zeitbudget des Hausarztes wurden häufig gewünscht (Tabelle 18).

Tabelle 18: Wünsche an den Hausarzt ( $n=258$; Mehrfachnennungen)

\begin{tabular}{lc}
\hline Wünsche $^{1)}$ & in \% \\
\hline Direkter und offener Umgang mit dem Thema & 56 \\
Information über mögliche Behandlung smethoden & 50 \\
Sich- Zeit- nehmen & 35 \\
Gegebenenfalls Überweisung an Spezialisten & 33 \\
Verläßlichkeit der ärztlichen Schweigepflicht & 24 \\
Sachlichkeit & 18 \\
Humor & 14 \\
\hline
\end{tabular}

\footnotetext{
${ }^{1)}$ Vorgegebene Liste
} 


\subsection{Einflüsse auf das Antwortverhalten}

Im diesem Abschnitt des Ergebnisteiles werden mögliche Einflußfaktoren auf die Antworten der Patienten vorgestellt. Hierzu zählen:

- $\quad$ Alter der Patienten (Kap. 5.4.1)

- $\quad$ Behandlungsdauer (Kap. 5.4.2)

- $\quad$ Kenntnisse des Hausarztes über persönliche Lebensumstände (Kap. 5.4.3)

- $\quad$ Vorliegen sexueller Probleme und sexuelle Zufriedenheit (Kap. 5.4.4)

- $\quad$ Sexualmedizinische Kenntnisse des Hausarztes (Kap. 5.4.5)

- Wohnort des Patienten (Kap. 5.4.6).

\subsubsection{Alter der Patienten}

Die Patientenstichprobe wurde in zwei Altersgruppen unterteilt (s. S. 19). Ein signifikanter Zusammenhang zwischen der Information der Ärzte über die persönlichen Lebensumstände der Patienten und ihrem Alter konnte ermittelt werden: Bei 94 \% der Patienten über 45 Jahre waren die Hausärzte mit über die persönlichen Lebensumstände informiert; in der jüngeren Vergleichsgruppe waren sie es bei 74 \% $(p=0,001)$.

Ebenso stand die Frage, ob der Hausarzt seine Bereitschaft, über Sexualprobleme zu sprechen, durch direktes Ansprechen während der Behandlung signalisieren solle, im Zusammenhang mit dem Alter der Patienten: Patienten über 45 Jahre fanden das eher angebracht als die jüngeren Männer (Tabelle 19).

Die Variable Alter hatte hingegen keinen Einfluß auf die Frage, ob der Hausarzt von sich aus das Thema Sexualstörungen ansprechen sollte oder nicht. 
Tabelle 19: Hausarzt soll Sexualprobleme während einer Behandlung direkt ansprechen $(n=273)$

\section{Direktes Ansprechen von Sexualproblemen}

Alter des Patienten

$\begin{array}{cc}\text { ja } & \text { nein } \\ n(\%) & n(\%)\end{array}$

$<=45$ Jahre

$85(57)$

$63(43)$

> 45 Jahre

$89(71)$

$36(29)$

alle

$174(64)$

$99(36)$

$\left(\mathrm{Chi}^{2}: 5,56 ; \mathrm{df}: 1 ; \mathrm{p}=0,018\right)$

Die Männer, die eher mit einem Arzt als mit einer Ärztin über Sexualprobleme sprechen würden, waren älter als die Patienten, für die das Geschlecht des Arztes keine Rolle spielte (Tabelle 20).

Tabelle 20: Bedeutung des Geschlechts des Hausarztes als potentieller Gesprächspartner bei sexuellen Problemen $(n=286)$

\section{Geschlecht des Hausarztes}

Alter des Patienten

männlich

$\mathrm{n}(\%)$

weiblich

$\mathrm{n}(\%)$

$\mathrm{n}(\%)$

$<=45$ Jahre

$>45$ Jahre

alle

$44(30)$

68 (49)

$112(39)$
8 ( 5)

$5(4)$

$13(5)$
$95(65)$

$66(47)$

$161(56)$

$\left(C h i^{2}: 10,8 ; d f: 2 ; p=0,004\right)$ 
Kein Zusammenhang bestand hingegen zwischen dem Alter der Patienten und ihrer sexuellen Zufriedenheit (Corr.: -0,05051 (Spearman); $p=0,4075$ )

Lustlosigkeit und Erektionsstörungen waren jedoch deutlich altersabhängig: je stärker die Patienten unter den genannten Schwierigkeiten litten, desto älter waren sie im Durchschnitt (Tabelle 21).

Tabelle 21: Sexualprobleme in Abhängigkeit vom Alter

\begin{tabular}{lccccc}
\hline & & \multicolumn{2}{c}{ Durchschnittliches Alter (Jahre) } & \\
\cline { 3 - 4 } & nie & selten & manchmal & häufig & immer \\
Lustlosigkeit & 39 & 43 & 44 & 54 & 46 \\
Erektionsstörungen & 39 & 43 & 53 & 60 & 67 \\
\hline
\end{tabular}

Erektionsstörungen: Corr.: 0,38 (Spearman); $p=0,0001$

Lustlosigkeit: Corr.: 0,18 (Spearman); $p=0,0049$

Ebenfalls ein signifikanter Zusammenhang bestand zwischen dem Alter und der Einschätzung der Ursachen für die sexuellen Probleme: Jüngere Männer gaben eher psychische Ursachen an (Tabelle 22).

Tabelle 22: Ursachen in Abhängigkeit vom Alter $(n=111)$

\section{Ursachen für die Sexualprobleme}

\section{Alter des Patienten}

$<=45$ Jahre

$>45$ Jahre

alle psychisch

$$
\text { n (\%) }
$$

$39(75)$

$30(51)$

$69(62)$ körperlich

n (\%)

$13(25)$

$29(49)$

42 (69)

(Chi': 10,$5 ; d f: 3 ; p<0,009)$ 


\subsubsection{Behandlungsdauer}

Erwartungsgemäß zeigte sich ein Zusammenhang zwischen der Behandlungsdauer und den Kenntnissen des Hausarztes über die persönlichen Lebensumstände des Patienten. Bei 93 \% der Männer, die sich schon seit mehr als fünf Jahren in hausärztlicher Behandlung befanden, war der Hausarzt über deren persönliche Lebensumstände informiert, dagegen nur bei $74 \%$ der Patienten, die erst kürzer in Behandlung waren $(p=0,001)$.

Von den Männern, die schon einmal ihren Hausarzt wegen sexueller Probleme konsultierten, waren 72 \% schon seit längerem bei ihrem Hausarzt in Behandlung, $28 \%$ dagegen erst seit weniger als fünf $(p=0,020)$.

\subsubsection{Persönliche Lebensumstände}

Auch zwischen der Bereitschaft, bei sexuellen Problemen den Hausarzt aufzusuchen, und der Kenntnis des Hausarztes über die persönlichen Lebensumstände dieser Patienten scheint ein Zusammenhang zu bestehen: von den 33 Patienten, die ihren Hausarzt wegen sexueller Probleme aufgesucht hatten, war der Hausarzt nur bei einem Patienten (3\%) nicht über seine persönlichen Lebensumstände informiert. In der Gruppe der übrigen Befragten $(n=250)$ meinten hingegen 46 Männer (18\%), daß der Hausarzt ihre persönlichen Lebensumstände nicht kenne.

Ähnlich fiel die Antwort auf die Frage aus, ob man bei Bedarf mit dem Hausarzt über Sexualschwierigkeiten sprechen könne: Wenn der Hausarzt über die Lebensumstände informiert war, glaubten deutlich mehr Männer mit ihrem Hausarzt über Sexualschwierigkeiten sprechen zu können, als im Falle mangelnder Information (Tabelle 23). 
Tabelle 23: Gesprächsbereitschaft der Patienten und Kenntnis des Hausarztes über die persönlichen Lebensumstände $(n=289)$

Gespräche über Sexualprobleme mit dem Hausarzt möglich

Hausarzt kennt Lebensumstände

ja

nein

alle

\section{ja}

n (\%)

$204(82)$

$24(48)$

$228(77)$ nein

n (\%)

44 (18)

$26(52)$

$70(23)$

$\left(\mathrm{Chi}^{2}: 27,1 ; \mathrm{df}: 1 ; \mathrm{p}=0,001\right)$

\subsubsection{Sexualstörungen und sexuelle Zufriedenheit}

Kein Zusammenhang konnte in der Befragung zwischen dem Vorliegen eines Sexualproblems und der sexuellen Zufriedenheit festgestellt werden (Tabelle 24):

Tabelle 24: Zufriedenheit mit dem Sexualleben in Abhängigkeit von dem Vorliegen sexueller Schwierigkeiten $(n=254)$

\section{Zufriedenheit mit dem Sexualleben}

Patient leidet unter einer oder mehreren Störungen

nein

ja

alle eher zufrieden

n (\%)

15 (79)

157 (67)

$172(68)$ eher unzufrieden

n (\%)

$4(21)$

78 (33)

82 (32)

(Chi $\left.{ }^{2}: 1,18 ; d f: 1 ; p=0,28\right)$ 
Männer, die von Sexualstörungen nicht betroffen waren, äußerten ebenso häufig den Wunsch, der Hausarzt solle seine Gesprächsbereitschaft durch direktes Ansprechen während einer Behandlung signalisieren (65\%) wie diejenigen Männer, die unter Sexualproblemen litten (64\%).

Auch die Häufigkeit des Vorkommens sexueller Störungen und die Frage, ob der Patient bei Bedarf gern mit dem Hausarzt über Sexualprobleme sprechen würde, hingen nicht zusammen. Gleiches gilt für die Frage, ob der Hausarzt das Thema Sexualprobleme während der Behandlung ansprechen soll. Jedoch hatten mehr Männer mit häufigen Sexualschwierigkeiten schon einmal ihren Hausarzt wegen dieser Probleme aufgesucht als die übrigen Patienten ( $28 \%$ bzw. 9\%; $p=0,001$ ).

\subsubsection{Einschätzung sexualmedizinischer Kenntnisse durch den Hausarzt}

Für die Frage, ob die ärztliche Einschätzung der eigenen sexualmedizinischen Kompetenz die Bereitschaft von Patienten beeinflußt, ihren Hausarzt wegen Sexualstörungen aufzusuchen, wurden die Antworten von Ärzten und Patienten pro Praxis in Beziehung gesetzt (nested design). Bezüglich der selbsteingeschätzten sexualmedizinischen Kenntnisse gab es einen leicht positiven Trend: so war der Anteil von Patienten, die bereits einmal wegen Sexualproblemen ihren Hausarzt aufgesucht hatten, etwas größer in den Praxen, in denen die Hausärzte ihre sexualmedizinische Kompetenz höher einstuften (rho: 0,26; $p=0,29$ ). Ähnliches gilt für Praxen, in denen die Ärzte die Bedeutung sexualmedizinischer Beratung höher einstuften. Dort hatten etwas mehr Patienten die Praxis bereits einmal wegen sexueller Probleme aufgesucht (rho: 0,$18 ; p=0,47$ ) und glaubten, dies auch bei Bedarf tun zu können (rho: 0,$13 ; p=0,62$ ). Allerdings waren in allen Fällen die Korrelationskoeffizjenten vergleichsweise niedrig und auch nicht signifikant. 


\subsubsection{Wohnort}

Der Wohnort beeinflußte weder die Antworten auf die Frage, ob der Hausarzt das Thema Sexualstörungen von sich aus ansprechen soll, noch auf die Frage, ob die Patienten bei Sexualproblemen tatsächlich ihren Hausarzt aufsuchten.

Eine gewisse Abhängigkeit zum Wohnort des Patienten fand sich bei der Frage, ob der Patient mit seinem Hausarzt bei Bedarf über sexuelle Schwierigkeiten sprechen könne: die meisten Patienten aus ländlichen Gebieten bejahten diese Frage (86 \%), etwas seltener (73\%) dagegen die „Städter“. 


\section{Diskussion}

\subsection{Zielsetzung der Studie}

Die vorliegende Studie erhob sexuelle Störungen und Probleme von Männern in hausärztlichen Praxen. Neben der Häufigkeit von Sexualschwierigkeiten waren auch die Wünsche männlicher Patienten an ihren Hausarzt bei Problemen im sexuellen Bereich Thema der Befragung. Parallel sollten berufliche Erfahrungen der Hausärzte mit dieser Problematik und ihre Vorgehensweise untersucht werden.

Diese Arbeit zielt nicht darauf ab, repräsentative Daten zur Prävalenz sexueller Dysfunktion bei Männern in Deutschland zu erheben. Vielmehr sollte eine erste „Bestandsaufnahme“ Rückschlüsse auf den Versorgungsbedarf hausärztlicher Patienten bei sexuellen Problemen zulassen.

\subsection{Beurteilung der Methode}

Der Einsatz von Fragebögen in unserer Studie war angemessen. Die Ärzte legten großen Wert auf Diskretion und geringe Arbeitsbelastung, was durch die Fragebogenerhebung möglich war. Auch die hohe Teilnahmebereitschaft der Patienten sowie die sehr hohe Prävalenzangabe bestätigt die Methode - gerade angesichts des noch immer tabuisierten Themas Sexualität. Es ist aber nicht auszuschließen, daß einige Fragen oder Verständnisschwierigkeiten möglicherweise ung eklärt blieben.

Die Einteilung der Zehner-Skalen in drei Kategorien dient der Anschaulichkeit der Daten, stellt jedoch kein validiertes Verfahren dar. Bei der Berechnung von Mittelwerten beispielsweise wurden aber die ursprünglichen Skalen ausgewertet.

Die Zusammenfassung von Dorf und Kleinstadt sowie größerer Stadt und Großstadt dient ebenfalls der besseren Übersichtlichkeit. 


\subsection{Die Stichprobe}

Ein Vergleich des Alters der teilnehmenden Patienten der vorliegenden Studie mit den Ergebnissen des ADT-Panels des Zentralinstituts für Kassenärztliche Versorgung (Kerek-Bodden et al. 2000) zeigt, daß die Altersgruppe der über 60-jährigen etwas unterrepräsentiert ist. Insbesondere gilt dies für die über 70-jährigen Männer, die mit nur $7 \%$ in der vorliegenden Studie, jedoch mit $15 \%$ in der zum Vergleich herangezogenen Studie vertreten sind. Möglicherweise ist dies ein Hinweis darauf, daß Praxen mit insgesamt älteren Patienten seltener teilnahmen als solche mit eher jüngeren. Außerdem könnte die Diskrepanz damit zusammenhängen, daß in die Ergebnisse des ADT-Panels auch Hausbesuche u.a. in Alten-und Pflegeheime miteinbezogen wurden, während diese, meist älteren Patienten, in der vorliegenden Studie nicht erfaßt wurden. Folglich sind Sexualprobleme bei Patienten in der Hausarztpraxis eher noch häufiger und/oder ausgeprägter als die Ergebnisse dieser Untersuchung zeigen, da mit zunehmendem Lebensalter erektile Dysfunktionen häufiger und schwerer werden (Feldman et al.1994). Ebenso verhält es sich mit der Häufigkeit von Appetenzstörungen (Laumann et al. 1999).

Hinsichtlich der Repräsentativität der Stichprobe ist auf die Teilnahmequote von $48 \%$ bei den Praxen hinzuweisen. Selektionseffekte sind nicht auszuschließen, d.h., daß möglicherweise vor allem Praxisinhaber an der Studie teilgenommen haben, die eine höhere Aufgeschlossenheit für Sexualprobleme zeigen. Bei der Beurteilung der Ergebnisse ist deshalb zu bedenken, daß Sexualität und Sexualprobleme vermutlich insgesamt noch seltener Thema in der Hausarztpraxis sein könnten, als diese Untersuchung zeigt. Einen solchen Zusammenhang vermuteten auch Broekman et al. (1994b).

Hausärzte, die eine Studienteilnahme ablehnten, nannten als Grund - neben Zeitmangel - am häufigsten die „Unzumutbarkeit“ des Themas für die Patienten. Dem steht jedoch die insgesamt hohe Teilnahmebereitschaft auf Seiten der Patienten in den übrigen Praxen (84 \%) gegenüber. Es steht zu befürchten, daß solche „Projektionen" die offene Kommunikation mit Patienten über Sexualprobleme eher behindern (Ittner et al. 1997b). 


\subsection{Häufigkeit von Sexualproblemen in der Hausarztpraxis}

Die Prävalenz sexueller Störungen und Probleme in der Hausarztpraxis war nesentlich höher als nach den bisherigen Forschungsergebnissen zu erwarten war: $93 \%$ der Patienten unserer Stichprobe gaben mindestens eine Sexualstörung an, unter der sie zumindest selten gelitten haben. Im Fragebogen wurde offengelassen, auf welchen Zeitraum sich die angegebenen Schwierigkeiten beziehen (s. Anhang). Nach Porst (1991) liegt die Prävalenz sexueller Störungen bei Männern in Arztpraxen bei $15-25 \%$. Read et al. (1997) fanden einen Anteil von $35 \%$ männlicher hausärztlicher Patienten, die an einer Form sexueller Dysfunktion litten; $75 \%$ der Männer äußerten sexuelle Unzufriedenheit. Auch die von Read befragten Patienten konnten ihre Probleme differenziert nach der Häufigkeit ihres Auftretens angeben. Nach einer ähnlichen Untersuchung in England (Dunn et al. 1998) hatten $34 \%$ der Männer in hausärztlicher Behandlung ein sexuelles Problem. Der Bezugszeitraum war jedoch auf die vergangenen drei Monate beschränkt.

Die vergleichsweise hohe Prävalenz sexueller Störungen in unserer Studie läßt sich zunächst damit erklären, daß die Patienten nach der Häufigkeit von Sexualproblemen (nie, selten, manchmal, häufig, immer) gefragt wurden. Selten oder manchmal zu antworten fällt leichter, als ein eindeutiges ja anzukreuzen. Es handelt sich auch nicht immer um ein aktuelles, sondern oft um ein sporadisch auftretendes Problem. So litt der größte Teil der Patienten in unserer Studie nur selten (21\%) oder manchmal (52 \%) unter Sexualstörungen. Unser Ergebnis ist aber wegen der ähnlich weitgefaßten Formulierung bei der Frage nach Sexualproblemen mit der Studie von Metz und Seifert (1990) vergleichbar: 97 \% der dort befragten Männer gaben an, im Laufe ihres Lebens schon einmal ein sexuelles Problem gehabt zu haben. Immerhin waren jedoch für ein Fünftel der Männer in unserer Stichprobe sexuelle Schwierigkeiten ein häufiges oder permanentes Problem. Zudem litten $42 \%$ der Patienten unter drei oder mehr Sexualproblemen, unabhängig von der Häufigkeit. Mit Bezug auf die dritte Hypothese ist davon auszugehen, daß in der Hausarztpraxis von einer Prävalenz sexueller Störungen im engeren Sinne (z.B. Erektionsstörungen) von ca. $30 \%$ auszugehen ist. Werden Sexualprobleme sehr weit gefaßt, ist mit einer höheren Prävalenz zu rechnen. 
Speziell unter Erektionsstörungen litten $32 \%$ der von uns befragten Männer Zumindest selten oder manchmal, $2 \%$ immer. Auch nach Rönsberg (1998) haben $35 \%$ der Männer gelegentliche und 1,6 \% permanente Erektionsstörungen. Wie bei Feldman et al. (1994) traten sowohl Erektionsstörungen als auch Lustlosigkeit in unserer Stichprobe mit zunehmendem Alter häufiger auf.

Bei der Bewertung der hohen Prävalenz sexueller Probleme in unserer Stichprobe ist zu berücksichtigen, daß nur ein relativ geringer Teil der Männer (17\%) mit ihrem Sexualleben sehr unzufrieden war und das Vorliegen von Sexualproblemen nicht mit sexueller Zufriedenheit korrelierte. Möglicherweise kommen nicht wenige Männer mit einer leichten Sexualstörung gut zurecht, ohne in Beziehungskonflikte zu geraten (Loewit 1996). Nicht jedes sexuelle Problem ist insofern auch zwingend „behandlungsbedürftig“. Umgekehrt sollten „harmlos“ erscheinende Sexualprobleme nicht als reine Befindlichkeitsstörungen bagatellisiert werden. Der Hausarzt steht vor der nicht leichten Entscheidung, ob ein artikuliertes Sexualproblem eine für den Patienten „behandlungsbedürftige“ Störung darstellt.

\subsection{Gespräche über Sexualprobleme und mögliche Gesprächsbarrieren}

Die vorliegende Studie bestätigt die Hypothese, daß nur ein geringer Teil der Patienten, die unter sexuellen Schwierigkeiten leiden, sich deshalb an ihren Hausarzt wendet (Hypothese 4). Trotz hoher Prävalenz hatte lediglich jeder zehnte Patient wegen sexueller Schwierigkeiten oder deren Folgen seinen Hausarzt aufgesucht. Auch in anderen Untersuchungen konsultierten Patienten mit sexuellen Problemen nur selten ihre Hausärzte (Metz und Seifert 1990). 79 \% der von uns befragten Männer glaubten jedoch, bei Bedarf problemlos mit inrem Hausarzt über sexuelle oder partnerschaftliche Schwierigkeiten sprechen zu können. Es ist nicht auszuschließen, daß Loyalität dem Arzt gegenüber diese Antwort induziert hat.

Ein Großteil der Patienten (83 \%) hielt ihre Hausärzte über ihre persönlichen Lebensumstände informiert; zudem waren $90 \%$ der befragten Männer seit mehr als einem Jahr (59\% schon über fünf Jahre) bei ihrem Arzt in Behandlung. Nach unseren Ergebnissen erleichtern Vertrautheit mit der Lebenssituation des Patienten so- 
wie ein länger bestehendes Arzt-Patienten-Verhältnis ein Gespräch über Sexualität (Hypothese 5); doch wurden Sexualprobleme nur selten in der Hausarztpraxis thematisiert. Vielleicht sind Sexualität und sexuelle Probleme nach wie vor ein Tabu (Burnett 1998; Holzapfel 1993; Levine 1992; Sanders 1993). Außerdem suchen Männer bei gesundheitlichen Problemen seltener und später ihren Hausarzt auf (Tudiver und Talbot 1999). Dies wird mit der sozialen Rolle des Mannes erklärt: von einem Mann wird erwartet „unverwundbar“ zu sein. Hilfe zu suchen ist kein akzeptiertes „männliches Verhalten“. Außerdem ist der erigierte Penis von jeher Symbol für Stärke und Männlichkeit (Gurguis 1998).

Bestätigt wurde unsere zweite Hypothese, daß Patienten mit ihrem Hausarzt über Sexualität und Sexualprobleme sprechen möchten, sie aber eine Gesprächsinitiative ihres Arztes begrüßen würden. Für 84 \% der Männer wäre es wichtig, bei Bedarf mit dem Hausarzt über Sexualprobleme sprechen zu können (Hypothese 1). $64 \%$ meinten, ihr Arzt könne seine Gesprächsbereitschaft z.B. durch direktes Ansprechen des Themas Sexualität bzw. Nachfragen während einer Behandlung sgnalisieren. Diese Patienten würden eine Gesprächsinitiative ihres Arztes nicht als Eingriff in die Intimsphäre sehen. Auch in anderen Studien betrachteten Patienten Sexualität und Sexualstörungen als angemessene Themen in der Hausarztpraxis (Ende et al. 1984; Metz und Seifert 1990; Read et al. 1997; Shahar et al. 1991) und bewerteten die hausärztliche Initiative als positiv (Metz und Seifert 1990).

Zusammenfassend läßt sich festhalten, daß Männer sich durchaus Gespräche mit ihrem Hausarzt bei Problemen im sexuellen Bereich wünschen, es in der Realität aber nur selten dazu kommt. Aufschlußreich sind daher Arztbefragungen.

Neben geringem sexualmedizinischen Wissen (47\%) und Zeitmangel (53 \%) betrachten Hausärzte auch Schamgefühle (32\%) als Grund, warum sie Sexualprobleme nicht ansprechen. Damit ist auch Hypothese 11 bestätigt. Auf Seiten der Patienten vermuteten die Hausärzte als vorrangige Gründe für ein Nicht-Ansprechen sexueller Schwierigkeiten Schamgefühle (90\%) und Formulierungsschwierigkeiten (74\%). 
Die Hausärzte in unserer Stichprobe gaben an, daß nur wenige Patienten primär wegen sexueller Probleme in ihre Praxis kamen (im Durchschnitt 7 pro Quartal). Dies deckt sich mit der Patientenbefragung: Nur wenige der von uns befragten Männer konsultierten bei Sexualstörungen ihren Hausarzt. Damit ist die Hypothese bestätigt, daß Sexualanamnesen in der hausärztlichen Praxis selten erhoben we rden (Hypothese 9). Allerdings ergaben sich bei durchschnittlich weiteren 16 Patienten pro Quartal während der Behandlung Beratungsanlässe zu sexuellen Themen. Durch problemorientiertes Nachfragen des Hausarztes ließen sich wohl noch häufiger sexuelle Probleme „aufdecken“. Dies gilt insbesondere auch für Patienten, die einem besonderen beruflichen Streß ausgesetzt sind - sahen doch $53 \%$ der befragten Patienten hier den Grund für ihre Sexualschwierigkeiten.

Hausärzte halten sexualmedizinische Kenntnisse für wichtig, schätzen ihre eigenen Kenntnisse jedoch eher gering ein - diese Hypothese konnte ebenfalls bestätigt werden (Hypothese 10). Sexualmedizinische Kenntnisse wie auch Sexualberatung bewerteten die Ärzte überwiegend als wichtig (53\% bzw. $56 \%$ ) oder sehr wichtig (47\% bzw. 28\%). Im Gegensatz hierzu sprachen sie ihre Patienten aber nur manchmal (53\%) oder selten (37\%) auf eventuell bestehende Sexualprobleme an. Lediglich $16 \%$ der befragten Ärzte versuchten dies grundsätzlich bei jeder Gesundheitsuntersuchung zu tun (Hypothese 9). Die in der Literatur beschriebene Unsicherheit der Ärzte aufgrund mangelnder Kenntnisse auf diesem Gebiet wurde von den befragten Hausärzten bestätigt (Broekman et al. 1994b; Holzapfel 1993; Sanders 1993; Baker 1991; Levine 1992). Dieser Zusammenhang wird zudem durch den mehrheitlich geäußerten Wunsch (78 \%) nach Fortbildungsveranstaltungen unterstrichen.

Vorbehalte auf Seiten der Hausärzte, Sexualprobleme in der Praxis nicht adäquat behandeln bzw. betreuen zu können, teilen Patienten nicht unbedingt. Diejenigen Patienten, die ihren Hausarzt schon einmal wegen sexueller Schwierigkeiten aufgesucht hatten, waren nach unseren Ergebnissen größtenteils mit der Behandlung sehr zufrieden (50\%) bis zufrieden (22\%). In der Tat lassen sich "leichtere" Beschwerden oft schon durch Informationen und Gespräche bessern (Holzapfel 1993; Kockott 1989; Moore und Goldstein 1980). Im Falle schwerwiegenderer sexueller 
Funktionsstörungen und notwendiger spezieller Therapieformen sind aber Spezialisten erforderlich (Suter 1998).

Bei Sexualproblemen sollten die Partner, die oft auch selbst Patienten beim Hausarzt sind, in die Behandlung miteinbezogen werden (Holzapfel 1993), da bei Sexualschwierigkeiten häufig auch Beziehungsprobleme bestehen (Loewit 1996). Tatsächlich versuchte dies etwa die Hälfte der von uns befragten Hausärzte zumindest manchmal; gelungen ist dies aber nur selten (47\%) oder nie (21\%). Das Einbeziehen der Partner in die Behandlung sexueller Dysfunktion ist wichtig (Hartmann 1998; Riley und Athanasiadis 1997), nicht nur für den Patienten, sondern auch für den Partner, der über das Problem genauso „verzweifelt“ sein kann (Kuritzky et al. 1998). Daß Sexualität nach wie vor ein Tabu darstellt, ist möglicherweise auch hier die Erklärung, warum die Partner nicht einbezogen werden.

\subsection{Einflußfaktoren auf die Gesprächsbereitschaft}

Ob ein Patient über Sexualprobleme spricht, hängt wesentlich davon ab, ob der Hausarzt seine persönlichen Lebensumstände kennt. Bestätigt ist damit die fünfte Hypothese. Männer, deren Hausarzt über ihre Lebensumstände gut informiert war, suchten ihren Hausarzt auch tendenziell häufiger wegen tatsächlich bestehender Sexualprobleme auf. Bei längerer Behandlungsdauer waren die Ärzte erwartungsgemäß besser über die Lebensumstände ihrer Patienten informiert. Männer, die seit mehr als fünf Jahren bei ihrem Hausarzt in Behandlung waren, suchten bei sexuellen Schwierigkeiten eher ihren Arzt auf.

Ein vergleichbarer Zusammenhang gilt für das Alter. Mehr ältere ( $>45$ Jahre) als jüngere Männer wünschten, daß der Hausarzt etwaige Probleme im sexuellen Bereich von sich aus ansprechen solle. Das hat möglicherweise damit zu tun, daß bei älteren Männern der Hausarzt besser über die persönlichen Lebensumstände seiner Patienten informiert war.

Inwieweit ärztliches Verhalten selbst und sexualmedizinisches Wissen eine Rolle für das Verhalten von Patienten spielen, konnte die vorliegende Untersuchung nur 
bedingt darstellen: es scheint so zu sein, daß sexualmedizinisches Wissen von Ärzten und die eingeschätzte Wertigkeit von Sexualberatung zumindest einen gewissen Einfluß auf das Konsultationsverhalten der Patienten haben - im Sinne einer Förderung bei entsprechenden ärztlichen Kenntnissen und Wertschätzug der Sexualmedizin.

Der Wohnort hatte Einfluß auf die Frage, ob Patienten bei Bedarf mit ihrem Hausarzt über sexuelle Schwierigkeiten sprechen könnten. Männer aus „ändlichen Pegionen" glaubten dies eher als Patienten aus der Stadt. Dies mag damit zusammenhängen, daß die Arzt-Patienten-Bindung in ländlichen Gegenden enger ist als in der Stadt (längere Behandlungsdauer, weniger Arztwechsel). Da aber in unserer Studie "Dorfbewohner“ ihren Hausarzt bei Sexualproblemen nicht öfter aufgesucht hatten als „Städter“, sollte der Einfluß des Wohnortes nicht überschätzt werden.

Die Hypothese, daß das Geschlecht des Arztes für die Mehrheit der Männer (56\%) keine Rolle spielte, wenn sie ein sexuelles Problem besprechen wollten, konnte bestätigt werden (Hypothese 6). Ein nicht unbeträchtlicher Teil der Männer bevorzugte aber einen „Geschlechtsgenossen“, wenn sie ein Sexualproblem hätten: nämlich $40 \%$ der Patienten aus Praxen mit einem Arzt und $32 \%$ der Patienten aus Praxen mit einer Ärztin. Ob diese Differenz von acht Prozentpunkten eine „Selbstselektion“ oder eine "Korrektur durch Erfahrung“ spiegelt, kann nicht entschieden werden. Ferner war jüngeren Männern das Geschlecht ihres Arztes bei der Thematik Sexualstörungen nicht so wichtig wie älteren Patienten, die eher einen Arzt bevorzugten.

\subsection{Medizinische Bedeutung der Sexualanamnese}

Die medizinische Relevanz einer Sexualanamnese betonen z.B. Ende et al. (1984). Sexualprobleme können

1. Krankheiten (z.B. Schlaflosigkeit und Depressionen) verursachen (Baker 1991; Shabsigh et al. 1998),

2. Hinweis auf eine noch nicht diagnostizierte Krankheit (z.B. Diabetes mellitus) sein und

3. durch therapeutische Maßnahmen (z.B. Medikamente) verursacht werden (Feldman et al. 1994). 
Die vorliegende Studie bestätigt die Hypothese, daß nach Meinung der Hausärzte Sexualstörungen im Zusammenhang mit in der Praxis häufig auftretenden Krankheiten und/oder verordneten Medikamenten stehen (Hypothese 7). $79 \%$ der Hausärzte gaben an, im Falle sexueller Schwierigkeiten eine Änderung der Medikation in Erwägung zu ziehen (Hypothese 8). 84 \% der Ärzte sahen psychosomatische Beschwerden als Anlaß, mit einem Patienten über mögliche Sexualprobleme zu sprechen. Auch Krankheiten wie Diabetes mellitus, Hypertonie, Prostataadenom oder Arteriosklerose würden viele Hausärzte veranlassen, ihre Patienten nach sexuellen Funktionsstörungen zu fragen. Gleiches gilt für Medikamente wie Antihypertonika, Antidepressiva und Diuretika, die sexuelle Funktionsstörungen zur Folge haben können. Diese positiven Ergebnisse müssen jedoch vor dem Hintergrund betrachtet werden, daß Gespräche über Sexualität insgesamt selten stattfinden.

$13 \%$ der befragten Patienten brachten ihre Sexualprobleme mit einer Krankheit, $9 \%$ mit Medikamenten in Verbindung. Ein Drittel der Männer war nach eigenen Angaben chronisch krank. Meist litten sie unter Herz-Kreislauf-Krankheiten bzw. Diabetes - beides wichtige Risikofaktoren für eine erektile Dysfunktion (Feldman et al. 1994). Jeder fünfte Patient nannte Schlafstörungen als Auswirkung der von inm geschilderten Sexualprobleme, jeder vierte Depressionen und Niedergeschlagenheit. Berichten Patienten derartige Beschwerden, so legen die Ergebnisse dieser Untersuchung nahe, in Zukunft häufiger nach Sexualschwierigkeiten zu fragen - ansonsten werden möglicherweise nur die Symptome (z.B Schlafstörungen) behandelt, und nicht ihre Ursache.

\subsection{Viagra $^{\circledR}$ in der Hausarztpraxis}

Sildenafil ist nach bisher vorliegenden Ergebnissen ein einfach zu handhabendes, wirksames und relativ gut verträgliches Medikament bei erektiler Dysfunktion (Morales et al. 1998; Goldstein et al. 1998). Einige Autoren äußerten jedoch Zweifel an der Unbedenklichkeit seines Einsatzes, da sie einen möglichen Mißbrauch als "Lifestyle-Droge“ und eine Verhinderung weitergehender physischer und/oder psychischer Diagnostik befürchten (Hengeveld 1998; Himmel 2000; Kochen 2000; Utiger 1998). Die Bereitschaft der Patienten selbst, sich vor Verordnung von Sildenafil 
eingehend untersuchen zu lassen, läßt nach Schaudig (1999) deutlich nach. Auch die Antworten der von uns befragten Ärzte spiegelten dieses breite Meinungsbild wider. $42 \%$ betonten, die Behandlung mit Sildenafil lenke von den eigentlichen psychischen Problemen des Patienten ab; 16 \% würden Sildenafil aufgrund der unerwünschten Wirkungen nur in schwersten Fällen erektiler Dysfunktion verordnen. Ein Drittel der Ärzte dagegen hält die Diskussion um Nebenwirkungen von Sildenafil in Anbetracht der starken psychischen Belastung von Patienten mit erektiler Dysfunktion für unangemessen. Ein Viertel glaubt, Erektionsprobleme könnten durch Sildenafil gelöst werden, bevor es zu gravierenden psychischen Schäden komme.

Unsere Hypothese, daß sich im Zusammenhang mit der Diskussion um Sildenafil häufiger Beratungsanlässe über Erektionsprobleme bzw. Sexualfragen bei Patienten ergeben, konnte bestätigt werden: bei fast jedem zweiten Hausarzt war dies der Fall (Hypothese 12). Nach Berghof (2000) suchten ein Jahr nach der Zulassung von Viagra ${ }^{\circledR}$ in Deutschland doppelt so viele Männer ihren Arzt wegen erektiler Dysfunktion auf wie vorher. Das Medikament wurde 500000 Männern in Deutschland verschrieben. Zwei Drittel der Hausärzte verordneten es bereits. Gerade durch Sildenafil kann der Hausarzt zunehmend Ansprechpartner bei sexualmedizinischen Fragen werden (Tiefer 1998).

Auch bei einer Behandlung mit Sildenafil ist die häufige Verknüpfung organischer und psychischer Ursachen zu beachten (Porst 1991) und sollten die Partner einbezogen werden (Utiger 1998). Die Kombination aus psychischer und physiologischer Betreuung führt in vielen Fällen zu besseren Ergebnissen (Hartmann 1998; Riley und Athanasiadis 1997; Tiefer und Schütz-Müller 1995) als bei „einseitiger“ Behandlung. 


\subsection{Ausblick}

Die vorliegende Untersuchung zeigt bei Männern einen hohen Bedarf nach sexualmedizinischer Betreuung durch ihren Hausarzt. Die Ergebnisse dieser Studie sollten Hausärzte dazu ermuntern, eine Sexualanamnese regelmäßig in die Basis- anamnese einzuschließen. Wünschenswert wäre, dem Thema Sexualität bereits im Studium (mehr) Raum zu geben, um möglichst schon dort Hemmungen und Probleme anzusprechen und abzubauen. Dies könnte angehende Ärzte für Sexualschwierigkeiten ihrer Patienten sensibilisieren.

Nicht geklärt wurde die Frage, was genau Patienten davon abhält, mit inrem Hausarzt über Sexualprobleme zu sprechen. Hier besteht Forschung sbedarf.

Wünschenswert wären weiterhin Untersuchungen, die das tatsächliche Patientenverhalten (Konsultationsbereitschaft) im Zusammenhang mit der tatsächlichen $æ$ xualmedizinischen Kompetenz ihrer Hausärzte explorieren. 


\section{Zusammenfassung}

Ausgangspunkt. Als sexuelle Dysfunktion sind Störungen im Ablauf des sexuellen Reaktionszyklus definiert, die von den Betroffenen als nachteilig empfunden we rden. Internationale Studien berichten eine hohe Prävalenz von Sexualproblemen bei männlichen Hausarztpatienten. Ursachen wie auch Folgen von Sexualschwierigkeiten können psychischer und physischer Art sein. Bei Sexualschwierigkeiten könnte der Hausarzt ein wichtiger Ansprechpartner sein.

Methode. Fachärzte für Allgemeinmedizin und Patienten in Hausarztpraxen in den Einzugsgebieten Hildesheim und Heiligenstadt wurden standardisiert per Fragebogen zu Sexualproblemen befragt.

Ergebnisse. 20 Praxen (43\%) nahmen an der Studie teil. 19 Ärzte (95\%) und 307 Männer (84 \%) beantworteten die Fragebögen. Die Prävalenz sexueller Störungen bei männlichen hausärztlichen Patienten war in unserer Studie hoch. Insgesamt gaben $93 \%$ der Patienten an, unter einer Störung zumindest selten zu leiden. Für $20 \%$ der Männer waren jedoch sexuelle Schwierigkeiten ein häufiges oder permanentes Problem. Am häufigsten wurden Lustlosigkeit (73\%) und Ejaculatio praecox (66 \%) genannt. $49 \%$ der Männer gaben mindestens drei Störungen an. Nur wenige Patienten suchten jedoch ihren Arzt auch wegen sexueller Schwierigkeiten auf. Viele Patienten (178/277) würden es begrüßen, wenn ihr Hausarzt von sich aus nach möglichen Sexualproblemen während einer Behandlung fragen würde. Patienten sprechen eher mit ihrem Hausarzt über Sexualstörungen, wenn er ihre persönlichen Lebensumstände gut kennt ( $82 \%$ vs $48 \%$ ). Das Geschlecht des Hausarztes spielte dabei kaum eine Rolle. Ärzte als auch Patienten brachten Sexualstörungen in Verbindung mit in der Hausarztpraxis häufigen Krankheitsbildern und Medikamenten. Hausärzte hielten sexualmedizinische Kenntnisse sowie Sexualberatung in der hausärztlichen Praxis zwar für wichtig, sprachen aber nur selten ihre Patienten auf mögliche Sexualprobleme an. Gründe waren Zeitmangel, unzureichende Kenntnisse und Schamgefühle. Die Diskussion um Viagra ${ }^{\circledR}$ führte nach Ansicht eines jeden zweiten Hausarztes zu häufigeren Beratungsanlässen zum Thema Sexualstörungen.

Schlußfolgerung. Die hohe Prävalenz von Sexualstörungen bei Männern macht auf den Bedarf nach sexualmedizinischer Betreuung durch den Hausarzt aufmerksam. Ärzte sollten eine Sexualanamnese regelmäßig durchführen. 
8. Anhang

\subsection{Arztfragebogen}

8.2 Arztfragebogen (Grundauszählung)

8.3 Patientenfragebogen

8.4 Patientenfragebogen (Grundauszählung) 
Sehr geehrte(r) Frau/Herr Kollege/in, im Rahmen unseres Forschungsprojektes zu den familienmedizinischen Aufgaben des Hausarztes soll eine Doktorarbeit Bedarf und Möglichkeiten sexualmedizinischer Betreuung durch den Hausarzt bei männlichen Patienten mit Sexualproblemen klären. Hierzu möchten wir Sie bitten, den folgenden Fragebogen auszufüllen, der sich ausschließlich auf Ihre männlichen Patienten bezieht. Selbstverständlich werden alle Ihre Angaben streng vertraulich behandelt. Für ausführlichere Anmerkungen nutzen Sie bitte die Rückseite des des Fragebogens!

Vielen Dank für Ihre Mitarbeit!

Wieviele Patienten kommen primär wegen sexueller Probleme zu Ihnen?

ca._Patienten/Quartal $\quad \square$ gar keine $\square$ weiß nicht

Ergeben sich in Ihrer Praxis bei der Behandlung Ihrer männlichen Patienten nebenbei auch manchmal Hinweise auf eine sexuelle Störung?

$\square$ ja, und zwar vor allem bei folgenden Krankheiten:

nein

Falls Sie die letzte Frage mit ,ja“ beantwortet haben, wie häufig ist dies in etwa der Fall?

bei ca. Patienten/Quartal

Bei wievielen Patienten vermuten Sie sexuelle Schwierigkeiten als den eigentlichen Grund für die Konsultation?

bei ca. Patienten /Quartal

Wie häufig sprechen Sie Ihre Patienten auf mögliche sexuelle Schwierigkeiten an?

$\square$ nie $\square$ selten $\square$ manchmal $\square$ oft $\square$ sehr oft

Bei welcher Gelegenheit sprechen Sie Patienten auf mögliche sexuelle Probleme an?

(Mehrfachnennungen möglich)

$\square$ im Zusammenhang mit Fragen zur Familienplanung

$\square$ bei Fragen zu HIV

$\square$ bei psychosomatischen Beschwerden

$\square$ grundsätzlich bei jeder Gesundheitsuntersuchung

$\square$ andere Gelegenheiten:

Gibt es typische Krankheiten, bei denen Sie Patienten auf mögliche sexuelle Schwierigkeiten ansprechen? (Mehrfachnennungen möglich)

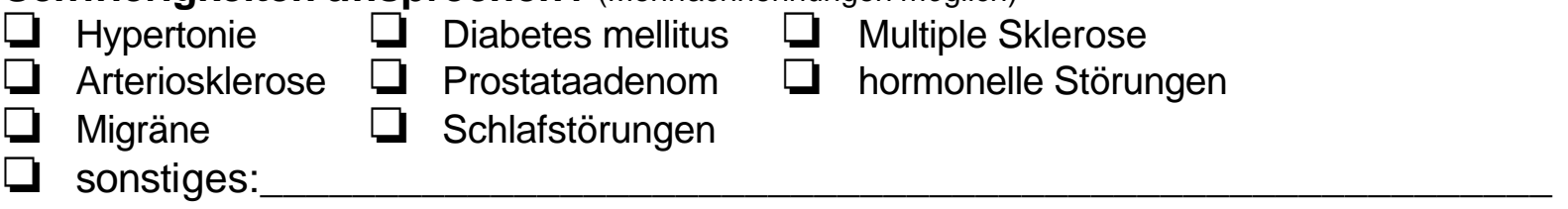

Gibt es typische Medikamente, bei denen Sie Ihre Patienten auf mögliche sexuelle Störungen ansprechen? (Mehrfachnennungen möglich)

$\square$ Antihypertonika $\square$ Diuretika $\square$ Psychopharmaka

$\square$ sonstige: 
Welche Gründe gibt es Ihrer Meinung nach für den Arzt, sexuelle Probleme in der hausärztlichen Praxis nicht anzusprechen? (Mehrfachnennungen möglich)
$\square$ Zeitmangel
unzureichende Kenntnisse auf diesem Gebiet
$\square$ Schamgefühle
$\square$ nicht daran zu denken
$\square$ Formulierungsschwierigkeiten
$\square$ sonstiges:

Welche Gründe gibt es Ihrer Meinung nach für Patienten, bei sexuellen Problemen ihren Hausarzt nicht zu Rate zu ziehen? (Mehrfachnennungen möglich)

$\begin{array}{ll}\square \text { Schamgefühle } & \square \text { mangelndes Vertrauen zum Arzt } \\ \square \text { Formulierungsschwierigkeiten } & \square \text { die Angst, den Arzt zu „enttäuschen“ } \\ \square \text { sonstiges: } & \end{array}$

Für wie wichtig halten Sie sexualmedizinische Kenntnisse in der hausärztlichen Praxis? (Kreuzen Sie bitte eine der Zahlen $1=u n w i c h t i g$ bis 10=sehr wichtig an!)
Unwichtig (1)
(2)
(3)
(4)
(5)
(6) (7)
(8)
(9) (10) sehr wichtig

Für wie wichtig halten Sie Sexualberatung in der hausärztlichen Praxis? (Kreuzen Sie bitte eine der Zahlen 1=unwichtig bis 10=sehr wichtig an!)
unwichtig (1)
(2)
(3)
(4) (5)
(6) (7)
(8)
(9)
(10) sehr wichtig

Wie beurteilen Sie Ihre eigenen sexualmedizinischen Kenntnisse?

(Kreuzen Sie bitte eine der Zahlen $1=$ sehr gering bis $10=$ sehr gut an)
sehr gering (1)
(2)
(3)
(4) (5)
(6) (7)
(8)
(9) (10) sehr gut

Glauben Sie, in Ihrer Praxis sexuelle Störungen behandeln zu können? (Mehrfachnennungen möglich)
Erektionsstörungen
- Libidostörungen
$\rightarrow$ Ejaculatio praecox
- Ejaculatio retardata
$\square \quad$ ja
ja $\square$ nein
$\square \quad$ ja $\square$ nein
D sonstige:

Wie behandeln Sie Patienten mit Sexualstörungen? (Mehrfachnennungen möglich)

$\square$ Medikationsänderung $\square$ Hormonsubstitution $\square$ Schwellkörperinjektion
$\square$ Sildenafil(Viagra $₫$ ) $\square$ Vakuumapparate $\quad \square$ Information und Gespräche
$\square$ Überweisung an Urologen $\square$ Überweisung an Psychotherapeuten
$\square$ sonstiges:

Wie oft versuchen Sie, die Partnerin oder den Partner des Patienten in die Behandlung mit einzubeziehen?

$\square$ immer $\square$ meistens $\square$ manchmal $\square$ selten $\square$ nie

Wie oft gelingt es, die Partnerin oder den Partner des Patienten mit einzubeziehen?

$\square$ immer $\square$ meistens $\square$ manchmal $\square$ selten $\square$ nie 
Was halten Sie von Sildenafil (Viagra ${ }^{\circledR}$ )? (Mehrfachnennungen möglich)

$\square$ Sildenafil (Viagra ${ }^{\circledR}$ ) ist ein gutes Mittel, um Potenzprobleme zu lösen, bevor es zu gravierenden psychischen Problemen kommt.

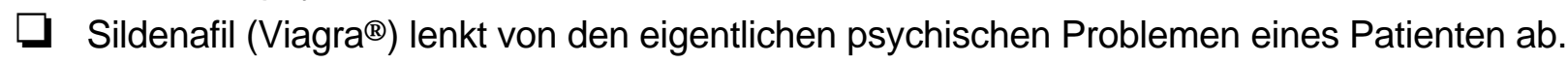

$\square$ Aufgrund der unerwünschten Wirkungen sollte Sildenafil (Viagra ${ }^{\circledR}$ ) nur in schwersten Fällen verordnet werden.

$\square$ In Anbetracht der starken psychischen Belastungen von Patienten mit erektiler Dysfunktion halte ich die Diskussion um Nebenwirkungen von Sildenafil (Viagra ${ }^{8}$ ) für unangemessen.

$\square$ sonstiges:

Haben sich im Zusammenhang mit der Diskussion um Sildenafil (Viagra ${ }^{\circledR}$ ) häufiger Beratungsanlässe über Potenzprobleme bzw. Sexualfragen bei Patienten ergeben?

$\square$ ja $\square$ nein $\square$ darauf habe ich nicht geachtet

Würden Sie Sildenafil (Viagra ®) verschreiben?

$\square$ ja $\square$ nein $\square$ weiß nicht

Würden Sie sich Fortbildungsveranstaltungen zum Thema „Sexualstörungen“ wünschen?

$\square$ ja, mich interessieren vor allem folgende Bereiche:

$\square$ nein

Zum Abschluß bitten wir Sie noch um einige Angaben zu Ihrer Person und zu Ihrer Praxis :

Ihr Alter: Jahre

Ihr Geschlecht: $\square$ männlich

$\square$ weiblich

Niedergelassen seit:

19

Größe des Praxisortes: $\square$ bis 5.000 Einwohner

$\square$ 5.000-20.000 Einwohner
$\square$ 20.000-100.000 Einwohner
$\square$ mehr als 100.000 Einwohner

Scheinzahl/Quartal:

$\square$ bis 500

$\square$ 500-999

$\square$ 2.000-2.499

$\square$ 1.500-1.999

$\square$ über 2.500

Art der Praxis: $\quad \square$ Einzelpraxis $\quad \square$ Gemeinschaftspraxis $\square$ Praxisgemeinschaft Zusatzbezeichnung(en): 


\section{Anhang 8.2: Grundauszählung Ärzte (Basis: $n=19$ )}

Wieviele Patienten kommen primär wegen sexueller Probleme zu Ihnen?

min.: 1; max.: 20 Patienten/Quartal

Ø: 7 Patienten/Quartal

Ergeben sich in Ihrer Praxis bei der Behandlung Ihrer männlichen Patienten nebenbei auch manchmal Hinweise auf eine sexuelle Störung?

$-\mathrm{ja}$

- nein$$
n=1(5 \%)
$$

Falls Sie die letzte Frage mit ,,ja“ beantwortet haben, wie häufig ist dies in etwa der Fall?

min.: 0; max.: 30 Patienten/Quartal Ø: 6 Patienten/Quartal

Bei wievielen Patienten vermuten Sie sexuelle Schwierigkeiten als den eigentlichen Grund für die Konsultation?

min.: 0; max.: 30 Patienten/Quartal Ø: 10 Patienten/Quartal

Wie häufig sprechen Sie Ihre Patienten auf mögliche sexuelle Schwierigkeiten an?

- nie

- selten

- manchmal

- oft

- sehr oft

$$
\begin{aligned}
& n=-(-\%) \\
& n=7(37 \%) \\
& n=10(53 \%) \\
& n=2(10 \%) \\
& n=-(-\%)
\end{aligned}
$$

Bei welcher Gelegenheit sprechen Sie Patienten auf mögliche sexuelle Probleme an?

(Mehrfachnennungen möglich)

- im Zusammenhang mit Fragen zur Familienplanung

- bei Fragen zu HIV

- bei psychosomatischen Beschwerden

- grundsätzlich bei jeder Gesundheitsuntersuchung

- andere Gelegenheiten

$\begin{array}{ll}n=10 & (53 \%) \\ n=9 & (47 \%) \\ n=16 & (84 \%) \\ n=3 & (16 \%) \\ n=4 & (21 \%)\end{array}$

Gibt es typische Krankheiten, bei denen Sie Patienten auf mögliche sexuelle Schwierigkeiten ansprechen?

(Mehrfachnennungen möglich)

- Hypertonie

- Diabetes mellitus

- Multiple Sklerose

- Arteriosklerose

- Prostataadenom

- hormonelle Störungen

- Migräne

- Schlafstörungen

- sonstiges

$$
\begin{array}{ll}
n=12 & (63 \%) \\
n=15 & (79 \%) \\
n=2 & (11 \%) \\
n=7 & (37 \%) \\
n=11 & (58 \%) \\
n=8 & (42 \%) \\
n=1 & (5 \%) \\
n=5 & (26 \%) \\
n=1 & (5 \%)
\end{array}
$$


Gibt es typische Medikamente, bei denen Sie Ihre Patienten auf mögliche sexuelle Störungen ansprechen? (Mehrfachnennungen möglich)

- Antihypertonika

- Diuretika

- Psychopharmaka

- sonstige

Welche Gründe gibt es Ihrer Meinung nach für den

Arzt, sexuelle Probleme in der hausärztlichen Praxis

nicht anzusprechen? (Mehrfachnennungen möglich)

- Zeitmangel

- unzureichende Kenntnisse auf diesem Gebiet

$n=10(53 \%)$

- Schamgefühle

- Formulierungsschwierigkeiten

$\mathrm{n}=9(47 \%)$

$n=6(32 \%)$

$\mathrm{n}=5(26 \%)$

- nicht daran zu denken

$\mathrm{n}=7(37 \%)$

- sonstiges

$n=5(26 \%)$

Welche Gründe gibt es Ihrer Meinung nach für

Patienten, bei sexuellen Problemen ihren Hausarzt

nicht zu Rate zu ziehen? (Mehrfachnennungen möglich)

- Schamgefühle

- mangelndes Vertrauen zum Arzt

- Formulierungsschwierigkeiten

- die Angst, den Arzt zu „enttäuschen“

- sonstiges

$\begin{array}{ll}n=17 & (90 \%) \\ n=4 & (21 \%) \\ n=14 & (74 \%) \\ n=3 & (16 \%) \\ n=1 & (5 \%)\end{array}$

Für wie wichtig halten Sie sexualmedizinische

Kenntnisse in der hausärztlichen Praxis?

(Kreuzen Sie bitte eine der Zahlen 1=unwichtig bis 10=sehr wichtig an!)

$$
\begin{aligned}
& -1 \\
& -(2) \\
& -3 \\
& -4 \\
& -4 \\
& -6 \\
& -7 \\
& -8 \\
& -9 \\
& -9
\end{aligned}
$$

$$
\begin{aligned}
& n=-(-\%) \\
& n=-(-\%) \\
& n=-(-\%) \\
& n=-(-\%) \\
& n=4(21 \%) \\
& n=2(11 \%) \\
& n=4(21 \%) \\
& n=5(26 \%) \\
& n=3(16 \%) \\
& n=1(5 \%)
\end{aligned}
$$


Für wie wichtig halten Sie Sexualberatung in der hausärztlichen Praxis?

(Kreuzen Sie bitte eine der Zahlen 1=unwichtig bis 10=sehr wichtig an!)

$$
\begin{aligned}
& -1 \\
& -(2) \\
& -3 \\
& -(4) \\
& -5 \\
& -(6) \\
& -7 \\
& -9 \\
& -9 \\
& -9 \\
& -(10)
\end{aligned}
$$

$$
\begin{aligned}
& \mathbf{n}=-(-\%) \\
& \mathbf{n}=-(-\%) \\
& \mathbf{n}=3(17 \%) \\
& \mathbf{n}=3(17 \%) \\
& \mathbf{n}=1(5 \%) \\
& \mathbf{n}=2(11 \%) \\
& \mathbf{n}=4(22 \%) \\
& \mathbf{n}=2(11 \%) \\
& n=3(17 \%) \\
& n=-(-\%)
\end{aligned}
$$

Wie beurteilen Sie Ihre eigenen sexualmedizinischen

\section{Kenntnisse?}

(Kreuzen Sie bitte eine der Zahlen 1=sehr gering bis $10=$ sehr gut an)

$$
\begin{aligned}
& -1 \\
& -2 \\
& -3 \\
& -(4) \\
& -5 \\
& -6 \\
& -7 \\
& -8 \\
& -9 \\
& -(10)
\end{aligned}
$$

$$
\begin{array}{ll}
n=-(-\%) \\
n=-(-\%) \\
n=1 & (5 \%) \\
n=-(-\%) \\
n=4 & (21 \%) \\
n=8 & (42 \%) \\
n=4 & (21 \%) \\
n=2 & (11 \%) \\
n=-(-\%) \\
n=-(-\%)
\end{array}
$$

Glauben Sie, in Ihrer Praxis sexuelle Störungen

\section{behandeln zu können?}

(Mehrfachnennungen möglich)

- Erektionsstörungen

- Libidostörungen

- Ejaculatio praecox

- Ejaculatio retardata

- sonstige

$$
\begin{array}{ll}
n=9 & (50 \%) \\
n=11 & (61 \%) \\
n=6 & (33 \%) \\
n=3 & (17 \%) \\
n=1 & (6 \%)
\end{array}
$$

Wie behandeln Sie Patienten mit Sexualstörungen?

(Mehrfachnennungen möglich)

- Medikationsänderung

- Hormonsubstitution

- Schwellkörperinjektion

- Sildenafil $\left(\right.$ Viagra $\left.^{\circledR}\right)$

- Vakuumapparate

- Information und Gespräche

- Überweisung an Urologen

- Überweisung an Psychotherapeuten

- sonstiges

$$
\begin{array}{ll}
n=15 & (79 \%) \\
n=9 & (47 \%) \\
n=2 & (11 \%) \\
n=10 & (53 \%) \\
n=- & (-\%) \\
n=11 & (58 \%) \\
n=14 & (74 \%) \\
n=6 & (32 \%) \\
n=2 & (11 \%)
\end{array}
$$


Wie oft versuchen Sie, die Partnerin oder den Partner

des Patienten in die Behandlung mit einzubeziehen?

- immer

- meistens

- manchmal

- selten

- nie

$$
\begin{array}{ll}
n=2 & (11 \%) \\
n=1 & (5 \%) \\
n=7 & (37 \%) \\
n=7 & (37 \%) \\
n=2 & (11 \%)
\end{array}
$$

Wie oft gelingt es, die Partnerin oder den Partner des Patienten mit einzubeziehen?

- immer

- meistens

- manchmal

- selten

- nie

$\begin{array}{ll}n=- & (-\%) \\ n=2 & (11 \%) \\ n=4 & (21 \%) \\ n=9 & (47 \%) \\ n=4 & (21 \%)\end{array}$

Was halten Sie von Sildenafil $\left(\right.$ Viagra $\left.^{\circledR}\right)$ ?

(Mehrfachnennungen möglich)

- Sildenafil (Viagra ${ }^{\circledR}$ ) ist ein gutes Mittel, um Potenzprobleme zu lösen, bevor es zu gravierenden psychischen Problemen kommt.

$n=5(26 \%)$

- Sildenafil $\left(\right.$ Viagra $\left.^{\circledR}\right)$ lenkt von den eigentlichen psychischen Problemen eines Patienten ab.

$n=8(42 \%)$

- Aufgrund der unerwünschten Wirkungen sollte Sildenafil (Viagra ${ }^{\circledR}$ ) nur in schwersten Fällen verordnet werden.

$n=3(16 \%)$

- In Anbetracht der starken psychischen Belastungen von Patienten mit erektiler Dysfunktion halte ich die Diskussion um Nebenwirkungen von Sildenafil $\left(\right.$ Viagra $^{\circledR}$ ) für unangemessen.

$$
n=4(21 \%)
$$

- sonstiges

$n=6(32 \%)$

Haben sich im Zusammenhang mit der Diskussion um Sildenafil $\left(\right.$ Viagra $^{\circledR}$ ) häufiger Beratungsanlässe über Potenzprobleme bzw. Sexualfragen bei Patienten ergeben?

- ja

- nein

- darauf habe ich nicht geachtet

$\mathrm{n}=9(47 \%)$

$n=10(53 \%)$

$\mathbf{n}=-(-\%)$

Würden Sie Sildenafil $\left(\right.$ Viagra $\left.^{\circledR}\right)$ verschreiben?

- ja

- nein

- weiß nicht

$\mathrm{n}=15(79 \%)$

$\mathrm{n}=1(5 \%)$

$n=3(16 \%)$

Würden Sie sich Fortbildungsveranstaltungen zum Thema „Sexualstörungen" wünschen?

$-\mathrm{ja}$

- nein

$n=14(78 \%)$

$\mathrm{n}=4(22 \%)$ 
Alter:

min.: 36; max.: 61 Jahre ø: 49 Jahre

\section{Geschlecht}

- männlich

$$
\begin{aligned}
& n=16(84 \%) \\
& n=3(16 \%)
\end{aligned}
$$

Niedergelassen seit

min.: 1; max.: 22 Jahre $\varnothing: 12$ Jahre

\section{Größe des Praxisortes}

- bis 5.000 Einwohner

- 5.000-20.000 Einwohner

$$
\mathbf{n}=-(-\%)
$$

$n=3(16 \%)$

- 20.000-100.000 Einwohner

$\mathrm{n}=4(21 \%)$

- mehr als 100.000 Einwohner

$n=12(63 \%)$

\section{ScheinzahI/Quartal}

- bis 500

-500-999

$-1.000-1.499$

$-1.500-1.999$

$-2.000-2.499$

- über 2.500

$\begin{array}{ll}n=1 & (6 \%) \\ n=8 & (44 \%) \\ n=5 & (28 \%) \\ n=3 & (17 \%) \\ n=1 & (6 \%) \\ n=- & (-\%)\end{array}$

\section{Art der Praxis}

- Einzelpraxis

- Gemeinschaftspraxis

- Praxisgemeinschaft

$n=15(79 \%)$
$n=4(21 \%)$
$n=-(-\%)$ 


\section{Sehr geehrter Patient,}

- die Abteilung Allgemeinmedizin der Universität Göttingen führt eine Studie über die familienmedizinischen Aufgaben des Hausarztes durch. Insbesondere möchten wir mehr darüber erfahren, wie männliche Patienten diese Aufgaben des Hausarztes beurteilen. Dazu brauchen wir Ihre Hilfe und möchten Sie bitten, den folgenden Fragebogen auszufüllen. Für ausführlichere Bemerkungen oder Anregungen nutzen Sie bitte die letzte Seite.

- Diese Untersuchung erfolgt vollkommen unabhängig von Ihrer Hausarztpraxis und die Fragebögen werden direkt an die Abteilung Allgemeinmedizin weitergeleitet. Selbstverständlich gilt absolute Schweigepflicht. Alle Ihre Angaben werden streng vertraulich behandelt.

- Bei der Angabe „Hausarzt“ ist auch „,Hausärztin“ gemeint.

Vielen Dank für Ihre Mitarbeit!

Weshalb suchen Sie heute Ihren Hausarzt auf? (Mehrfachnennungen möglich)

weil ich akut krank bin (z.B. Grippe, Schnupfen, Halsentzündung)

wegen einer chronischen Erkrankung (z.B. Herzleiden, Hochdruck, Diabetes)

$\bigcirc$ wegen einer Vorsorgeuntersuchung (z.B. Krebsvorsorge)

$\bigcirc$ um mich zu einem gesundheitlichen Thema beraten zu lassen (z.B. Ernährungsfragen)

$\bigcirc$ sonstiges:

Seit wann sind Sie bei Ihrem Hausarzt in Behandlung?

O seit Jahr/en

Ist Ihr Hausarzt über Ihre persönlichen Lebensumstände (Beruf, Wohnsituation, familiäre Verhältnisse) informiert?

$\bigcirc \mathrm{ja}$, weil ich inm von mir aus darüber berichtet habe

$O \mathrm{ja}$, weil er danach gefragt hat

$\bigcirc \mathrm{ja}$, das hat sich so ergeben

$O$ nein, das geht inn nichts an

nein, weil ich darüber mit inm nicht sprechen kann

nein, weil ich denke, daß er darüber nicht sprechen möchte

sonstiges:

Glauben Sie, daß Sie - bei Bedarf - mit Ihrem Hausarzt über sexuelle oder partnerschaftliche Schwierigkeiten sprechen können?

$\bigcirc$ ja, das wäre problemlos möglich

$\bigcirc$ das wäre schwierig, weil ich glaube, daß er nicht genügend Zeit hat

$\bigcirc$ das wäre schwierig, weil ich das Thema zu persönlich finde

sonstiges:

Wäre es Ihnen wichtig, - bei Bedarf - mit Ihrem Hausarzt diese Probleme besprechen zu können?

$\bigcirc$ ja $\bigcirc$ nein $\bigcirc$ sonstiges:

Sollte ein Hausarzt von sich aus mögliche Schwierigkeiten in Sexualität und Partnerschaft zur Sprache bringen?

$\bigcirc$ ja, auf jeden Fall

$\bigcirc$ nein, es wäre an mir, diesen ersten Schritt zu tun 
Wie könnte Ihr Hausarzt signalisieren, daß er auch über sexuelle und partnerschaftliche Probleme zu sprechen bereit ist? (Mehrfachnennungen möglich)

$\bigcirc$ durch Auslegen von Informationsbroschüren und/oder Bücher zum Thema im Wartezimmer

durch direktes Ansprechen bzw. Nachfragen während einer Behandlung

sonstiges:

Glauben Sie, über sexuelle/partnerschaftliche Schwierigkeiten eher mit einem Arzt oder mit einer Ärztin sprechen zu können?

$\bigcirc$ eher mit einem Arzt

$\bigcirc$ eher mit einer Ärztin

$\bigcirc$ das Geschlecht spielt für mich keine Rolle

Im folgenden möchten wir Sie bitten, uns einige Fragen zum Thema Sexualität und Sexualstörungen zu beantworten. Füllen Sie diesen Teil des Fragebogens bitte auch dann aus, wenn Sie selbst von der Thematik nicht betroffen sind, weil auch die Angaben Nicht-Betroffener für die Auswertung unserer Studie wichtig sind.

Wie zufrieden sind Sie mit Ihrem Sexualleben? (Kreuzen Sie bitte eine der Zahlen $1=s e h r$ unzufrieden bis $10=$ sehr zufrieden an!)
sehr unzufrieden (1)
(2) (3) (4)
(5)
(6) (7)
(8)
(9) (10) sehr zufrieden

Es gibt eine Vielzahl von sexuellen Problemen, unter denen Männer leiden können. Wie ist das bei Ihnen? (Mehrfachnennungen möglich) Ich leide unter:
Lustlosigkeit
Onie $O_{\text {selten }}$
manchmal
Ohäufig
Oimmer
Versagensängsten
Onie
Oselten Omanchmal
Ohäufig
Oimmer
Erektionsstörungen
Onie
vorzeitigem Samenerguß
nie
Onie
Oselten
Omanchmal
Ohäufig
Oimmer
verzögertem Samenerguß
Onie
Oselten
Omanchmal
Ohäufig
Oimmer
ausbleibendem Samenerguß
Oselten Omanchmal
Ohäufig
Oimmer
Schmerzen beim Geschlechtsverkehr $\mathrm{Onie}_{\text {nie }}$
Fehlbildung des Penis
Oselten Omanchmal
Ohäufig
Oimmer
zu kleinem Penis
Oja Oetwas
Onäufig
Oimmer

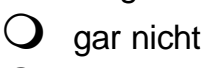
Oja $\quad O_{\text {etwas }}$
gar nicht

anderen Problemen:

Seit wann leiden Sie unter den oben genannten Schwierigkeiten?
$O$ erst seit dem letzten Jahr
$O$ seit Jahren
$O$ schon immer
$\mathrm{O}$ ich weiß nicht

Glauben Sie, daß die sexuellen Schwierigkeiten bei Ihnen eher seelische oder körperliche Ursachen haben?
$O$ eher seelische
eher körperliche
$\bigcirc$ ich weiß nicht 
Welche konkreten Ursachen haben oder hatten Ihre Probleme Ihrer Meinung nach? (Mehrfachnennungen möglich)

Hemmungen aufgrund von strenger Sexualerziehung

Schwierigkeiten in der Partnerschaft

unerfüllter Kinderwunsch

Erwartungsdruck von mir

Erwartungsdruck von anderen

$O$ beruflicher Streß

eine Krankheit, nämlich:

Medikamente, nämlich:

Haben oder hatten die sexuellen Probleme Auswirkungen für Sie? (Mehrfachnennungen möglich)

$\mathrm{O}$ sexuelle Unzufriedenheit

Schwierigkeiten in der Partnerschaft

Niedergeschlagenheit

Minderwertigkeitsgefühle

sonstiges:

Schlafstörungen

$\bigcirc$ Depressionen $\quad O$ keine

Gibt es jemanden, an den Sie sich - bei Bedarf wenden können, um über sexuelle Fragen und Probleme zu sprechen? (Mehrfachnennungen möglich)
O Partner/in
$O$ Freunde
Beratungsstelle
Selbsthilfegruppe
Urologe/in
Hausarzt/Hausärztin
niemanden
O Pastor/Pfarrer
sonstige:

Sind Sie wegen sexueller Probleme oder deren Folgen schon einmal bei Ihrem Hausarzt gewesen?
$\bigcirc$ ja
nein

Wenn ja, wie wurden Sie dann behandelt/betreut? (Mehrfachnennungen möglich)

$\bigcirc$ wir haben die Probleme ausführlich besprochen

mir wurden Medikamente verschrieben

$\bigcirc$ ich wurde an Spezialisten überwiesen

sonstiges:

Wie zufrieden waren Sie mit der Behandlung/Betreuung Ihrer Sexualprobleme durch Ihren Hausarzt? (Kreuzen Sie bitte eine der Zahlen 1=sehr unzufrieden bis 10=sehr zufrieden an!)
sehr unzufrieden (1)
(2)
(3) (4)
(5)
(6) (7)
(8)
(9)
(10) sehr zufrieden

Was würden Sie sich von Ihrem Hausarzt bei möglichen sexuellen Störungen wünschen vor allem wünschen? (Bitte nur die beiden wichtigsten angeben!)

$\bigcirc \mathrm{daß}$ er mich über mögliche Behandlungsmethoden informiert

$\bigcirc \mathrm{daß}$ er mich gegebenenfalls an einen Spezialisten überweist

daß er direkt und offen mit mir spricht

daß er sachlich bleibt

$\bigcirc$ daß er mir mit Humor über eventuelle Verlegenheiten hinweghilft

daß er sich Zeit nimmt

$\bigcirc$ daß ich mich voll auf seine ärztliche Schweigepflicht verlassen kann 
Bitte beantworten Sie uns zum Schluß noch einige Fragen zu Ihrer Person!

Alter: Jahre

\section{Schulbildung}

$O$ ohne Abschluß

mittlere Reife

Hauptschulabschluß

Abitur

Fach-/Hochschulabschluß

\section{Beruf}

$\mathrm{O}$ in Ausbildung/Studium

$\bigcirc$ nicht berufstätig/arbeitslos

berufstätig als:

Rentner

\section{Familienstand}

$\bigcirc$ ich bin verheiratet/lebe in eheähnlicher Gemeinschaft, seit :

$O$ ich habe einen festen Partner, seit :

$O$ ich bin alleinstehend, seit :

$O$ ich bin geschieden, seit :

$\mathrm{O}$ ich bin verwitwet, seit :

\section{Kinder}

$O$ ich habe Kind(er)

$\mathrm{O}$ ich habe kein Kind, obwohl ich mir seit

$O$ ich habe kein Kind, weil ich keines will Jahren eines wünsche

\section{Wohnort}

Dorf (unter 5.000 Einwohner)

Kleinstadt (5.000-20.000 Einwohner)

größere Stadt (20.000-100.000 Einwohner)

O Großstadt (mehr als 100.000 Einwohner)

\section{Leiden Sie an einer chronischen Krankheit?}

nein

ja, und zwar:

Hier haben Sie Platz für eigene Anmerkungen oder Ergänzungen: 


\section{Anhang 8.4: Grundauszählung Patienten (Basis: $\mathbf{n}=307$ )}

Weshalb suchen Sie heute Ihren Hausarzt auf?

(Mehrfachnennungen möglich)

- akute Krankheit

- chronische Erkrankung

- Vorsorgeuntersuchung

- Beratung

- sonstiges

$$
\begin{aligned}
& n=133(45 \%) \\
& n=120(40 \%) \\
& n=41 \quad(14 \%) \\
& n=25(8 \%) \\
& n=55(18 \%)
\end{aligned}
$$

Seit wann sind Sie bei Ihrem Hausarzt in Behandlung?

min.: 0 Jahre; max.:41 Jahre

Ist Ihr Hausarzt über Ihre persönlichen Lebensumstände

(Beruf, Wohnsituation, familiäre Verhältnisse) informiert?

- ja, von mir aus darüber berichtet

- ja er hat danach gefragt

- ja hat sich ergeben

- nein geht inn nichts an

- nein ich kann darüber nicht mit inm sprechen

- nein ich denke, daß er darüber nicht sprechen möchte

- sonstiges

$\begin{array}{rr}n=151 & (51 \%) \\ n=66 & (22 \%) \\ n= & 90 \\ n=15 & (50) \\ n= & (5 \%) \\ n= & (2 \%) \\ n= & 9(3 \%) \\ n= & 23(8 \%)\end{array}$

Glauben Sie, daß Sie - bei Bedarf - mit Ihrem Hausarzt über sexuelle oder partnerschaftliche Schwierigkeiten sprechen können?

- ja problemlos

- schwierig, weil ich glaube, daß er nicht genügend Zeit hat

- schwierig, weil ich das Thema zu persönlich finde

- sonstiges

Wäre es Ihnen wichtig, - bei Bedarf - mit Ihrem Hausarzt diese Probleme besprechen zu können?

- ja

- nein

$n=47(16 \%)$

Sollte ein Hausarzt von sich aus mögliche Schwierigkeiten in Sexualität und Partnerschaft zur Sprache bringen?

- ja

- nein

$n=133(45 \%)$

$n=162(55 \%)$

Wie könnte Ihr Hausarzt signalisieren, daß er auch über sexuelle und partnerschaftliche Probleme zu sprechen bereit ist?

(Mehrfachnennungen möglich)

- Informationsbroschüren und/oder Bücher zum Thema im Wartezimmer

- direktes Ansprechen bzw. Nachfragen

- sonstiges 
Glauben Sie, über sexuelle/partnerschaftliche Schwierigkeiten eher mit einem Arzt oder mit einer Ärztin sprechen zu können?

- Arzt

- Ärztin

- Geschlecht egal

$$
\begin{aligned}
& n=114(39 \%) \\
& n=13(5 \%) \\
& n=164(56 \%)
\end{aligned}
$$

Wie zufrieden sind Sie mit Ihrem Sexualleben?

(Kreuzen Sie bitte eine der Zahlen 1=sehr unzufrieden bis 10=sehr zufrieden an!)

$$
\begin{aligned}
& -1 \\
& -(2) \\
& -3 \\
& -(4) \\
& -5 \\
& -6 \\
& -8 \\
& -8 \\
& -9 \\
& -9 \\
& -(10)
\end{aligned}
$$

\begin{tabular}{|c|c|c|c|c|c|}
\hline & \begin{tabular}{|l} 
Nie \\
n (\%)
\end{tabular} & $\begin{array}{c}\text { Selten } \\
\mathrm{n}(\%)\end{array}$ & $\begin{array}{l}\text { Manchmal } \\
\text { n (\%) }\end{array}$ & $\begin{array}{l}\text { Häufig } \\
\text { n (\%) }\end{array}$ & $\begin{array}{l}\text { Immer } \\
\text { n (\%) }\end{array}$ \\
\hline Lustlosigkeit & $66(27)$ & $83(34)$ & $82(34)$ & $8(3)$ & $4(2)$ \\
\hline Versagensängste & $109(50)$ & $69(32)$ & $29(13)$ & $11(5)$ & $0(0)$ \\
\hline Erektionsstörungen & $136(62)$ & $49(22)$ & $23(10)$ & $9(4)$ & $4(2)$ \\
\hline Ejaculatio praecox & $74(34)$ & $56(26)$ & $63(29)$ & $23(10)$ & $2(1)$ \\
\hline Ejaculatio retardata & $101(51)$ & $65(33)$ & $25(12)$ & $7(3)$ & $1(1)$ \\
\hline $\begin{array}{l}\text { Ausbleibender } \\
\text { Samenerguß }\end{array}$ & $153(75)$ & $32(16)$ & $15(7)$ & $2(1)$ & $1(1)$ \\
\hline $\begin{array}{l}\text { Schmerzen beim } \\
\text { Geschlechtsverkehr }\end{array}$ & $168(83)$ & $22(11)$ & $11(5)$ & $1(1)$ & $0(0)$ \\
\hline & & $\begin{array}{c}j a \\
\mathrm{n}(\%)\end{array}$ & \multicolumn{2}{|c|}{$\begin{array}{l}\text { etwas } \\
\text { n (\%) }\end{array}$} & $\begin{array}{c}\text { gar nicht } \\
\text { n (\%) }\end{array}$ \\
\hline $\begin{array}{l}\text { Fehlbildungen des } \\
\text { Penis }\end{array}$ & \multicolumn{2}{|r|}{$0(0)$} & \multicolumn{2}{|l|}{$12(6)$} & $180(94)$ \\
\hline Zu kleiner Penis & & $3(2)$ & \multicolumn{2}{|c|}{$26(14)$} & $159(84)$ \\
\hline
\end{tabular}

$$
\begin{aligned}
& n=18(7 \%) \\
& n=10(4 \%) \\
& n=19(7 \%) \\
& n=16(6 \%) \\
& n=25(9 \%) \\
& n=24(9 \%) \\
& n=37(14 \%) \\
& n=49(18 \%) \\
& n=26(10 \%) \\
& n=51(19 \%)
\end{aligned}
$$

Es gibt eine Vielzahl von sexuellen Problemen, unter denen Männer leiden können. Wie ist das bei Ihnen? (Mehrfachnennungen möglich) 
Seit wann leiden Sie unter den oben genannten

Schwierigkeiten?

min.:0 Mon.; max.: $32 \mathrm{~J}$.

Glauben Sie, daß die sexuellen Schwierigkeiten bei Ihnen

eher seelische oder körperliche Ursachen haben?

- seelische

- körperliche

$n=70(34 \%)$

- weiß nicht

$\mathrm{n}=42(31 \%)$

$n=91(45 \%)$

Welche konkreten Ursachen haben oder hatten Ihre Probleme

Ihrer Meinung nach? (Mehrfachnennungen möglich)

- Hemmungen aufgrund von strenger Sexualerziehung

- Schwierigkeiten in der Partnerschaft

- unerfüllter Kinderwunsch

- Erwartungsdruck von mir

- Erwartungsdruck von anderen

- beruflicher Streß

- eine Krankheit

- Medikamente

$$
\begin{aligned}
& n=16(8 \%) \\
& n=38(19 \%) \\
& n=7(3 \%) \\
& n=56(28 \%) \\
& n=16(8 \%) \\
& n=107(53 \%) \\
& n=26(13 \%) \\
& n=18(9 \%)
\end{aligned}
$$

Haben oder hatten die sexuellen Probleme Auswirkungen

für Sie? (Mehrfachnennungen möglich)

- sexuelle Unzufriedenheit

- Minderwertigkeitsgefühle

- Schwierigkeiten in der Partnerschaft

- Schlafstörungen

- Niedergeschlagenheit

- Depressionen

- sonstiges

$\begin{array}{rr}n= & 47(18 \%) \\ n=17 & (7 \%) \\ n= & 35(14 \%) \\ n= & 36(14 \%) \\ n= & 32(13 \%) \\ n=14(5 \%) \\ n=2(1 \%)\end{array}$

Gibt es jemanden, an den Sie sich - bei Bedarf wenden können, um über sexuelle Fragen und Probleme zu sprechen?

(Mehrfachnennungen möglich)

- Partner/in

- Freunde

- Beratungsstelle

- Selbsthilfegruppe

- Urologe/in

- Hausarzt/Hausärztin

- Pastor/Pfarrer

- sonstige:

$\begin{array}{lr}n=171 & (69 \%) \\ n=71 & (28 \%) \\ n= & 3(2 \%) \\ n= & 1(1 \%) \\ n= & 30(12 \%) \\ n= & 91(37 \%) \\ n= & 4(2 \%) \\ n= & 7(3 \%)\end{array}$

Sind Sie wegen sexueller Probleme oder deren Folgen schon einmal bei Ihrem Hausarzt gewesen?

- ja

$n=34(12 \%)$

- nein

$n=254(88 \%)$ 
Wenn ja, wie wurden Sie dann behandelt/betreut?

(Mehrfachnennungen möglich)

- wir haben die Probleme ausführlich besprochen

- mir wurden Medikamente verschrieben

- ich wurde an Spezialisten überwiesen

- sonstiges

- nicht zutreffend

$$
\begin{aligned}
& n=19(7 \%) \\
& n=10(4 \%) \\
& n=8(3 \%) \\
& n=-(-\%) \\
& n=254(88 \%)
\end{aligned}
$$

Wie zufrieden waren Sie mit der Behandlung/Betreuung Ihrer Sexualprobleme durch Ihren Hausarzt?

(Kreuzen Sie bitte eine der Zahlen 1=sehr unzufrieden bis 10=sehr zufrieden an!)

$$
\begin{aligned}
& -(1) \\
& -(2) \\
& -(3) \\
& -(4) \\
& -(5) \\
& -(6) \\
& -7 \\
& -8 \\
& -(9) \\
& -(10) \\
& \text { - nicht zutreffend }
\end{aligned}
$$

$$
\begin{aligned}
& n=3(1 \%) \\
& n=2(1 \%) \\
& n=4(1 \%) \\
& n=2(1 \%) \\
& n=1(1 / 2 \%) \\
& \mathrm{n}=-(-\%) \\
& n=4(1 \%) \\
& \mathrm{n}=7(3 \%) \\
& n=2(1 \%) \\
& \mathrm{n}=7(3 \%) \\
& n=253(89 \%)
\end{aligned}
$$

Was würden Sie sich von Ihrem Hausarzt bei möglichen sexuellen Störungen wünschen vor allem wünschen?

- Information über Behandlungsmethoden

$$
\begin{aligned}
& n=128(50 \%) \\
& n=85(33 \%) \\
& n=145(56 \%) \\
& n=46(18 \%) \\
& n=37(14 \%) \\
& n=89(35 \%) \\
& n=63(24 \%)
\end{aligned}
$$

\section{Alter}

\begin{tabular}{cc} 
Alter; Jahre & $\mathrm{n}(\%)$ \\
\hline $18-20$ & $13(4)$ \\
$20-29$ & $41(14)$ \\
$30-39$ & $62(21)$ \\
$40-49$ & $64(22)$ \\
$50-59$ & $53(18)$ \\
$60-69$ & $45(15)$ \\
$70-79$ & $18(6)$ \\
$80-84$ & $2(1)$
\end{tabular}


Schulbildung

- ohne Abschluß

- Hauptschulabschluß

$n=12(4 \%)$

- mittlere Reife

- Abitur

- Fach-/Hochschulabschluß

$n=98(33 \%)$

$\mathrm{n}=91(31 \%)$

$n=46(15 \%)$

$n=50(17 \%)$

\section{Beruf}

- in Ausbildung/Studium

$n=31(10 \%)$

- Arbeiter

- Angestellter

$n=60(20 \%)$

$\mathrm{n}=89(30 \%)$

- Beamter

$n=13(5 \%)$

- Selbständiger

$\mathrm{n}=8(3 \%)$

- arbeitslos

- Rentner

- berufstätig ohne nähere Bezeichnung

$\mathrm{n}=22(7 \%)$

$n=70(24 \%)$

$n=4(1 \%)$

\section{Familienstand}

- verheiratet/in eheähnlicher Gemeinschaft

$n=195(64 \%)$

- feste Partnerschaft

- alleinstehend

- geschieden

- verwitwet

$\mathrm{n}=65(21 \%)$

$n=37(12 \%)$

$n=5(2 \%)$

$n=3(1 \%)$

\section{Kinder}

- ich habe Kind(er)

min.: 1; max.: 6

- kein Kind

$\mathrm{n}=72(26 \%)$

- kein Kind trotz Kinderwunsch

$n=18(6 \%)$

\section{Wohnort}

- Dorf (unter 5.000 Einwohner)

- Kleinstadt (5.000-20.000 Einwohner)

- größere Stadt (20.000-100.000 Einwohner)

$n=28(9 \%)$

$n=48(16 \%)$

$\mathrm{n}=90(30 \%)$

- Großstadt (mehr als 100.000 Einwohner)

$n=133(45 \%)$

Leiden Sie an einer chronischen Krankheit? 


\section{Literaturverzeichnis}

Ahlen van H, Hertle L: Störungen der Samendeposition; in: Andrologie. Grundlagen und Klinik der reproduktiven Gesundheit des Mannes; hrsg. v. Nieschlag E, Behre HM; Springer Verlag, Berlin 1996, 183-215

Baier D, Philipp M (1994): Die Beeinflussung sexueller Funktionen durch Antidepressiva. Fortschr Neurol Psychiatr $\underline{62}, 14-21$

Baker M (1991): A GP's view of sexual dysfunction and its treatment. Med J Aust $\underline{155}, 62-64$

Berghof H, Pfizer GmbH, Karlsruhe, Abteilung Medizin: persönliche Mitteilung, 2000

Beutel M (1999): Psychosomatic aspects in the diagnosis and treatment of erectile dysfunction. Andrologia 31, 37 - 44

Britt H, Bhasale A Miles DA, Meza A, Sayer GP, Angelis M (1996): The sex of the general practitioner: a comparison of characteristics, patients and medical conditions managed. Med Care $\underline{34}, 403-415$

Broekman CPM, van der Werff ten Bosch JJ, Slob AK (1994a): The patient with erection problems and his general practitioner. Int $\mathrm{J}$ Impot Res $\underline{6}, 59-65$

Broekman CPM, van der Werff ten Bosch JJ, Slob AK (1994b): An investigation into the management of patients with erection problems in general practice. Int $\mathrm{J}$ Impot $\operatorname{Res} \underline{6}, 67-72$

Buddeberg C (1994): Sexualanamnese, Sexualberatung, Sexualtherapie. Ther Umsch 51, $104-108$

Buddeberg C: Sexualberatung. Eine Einführung für Ärzte, Psychotherapeuten und Familienberater. 3. Auflage; Enke Verlag, Stuttgart 1996 
Burnett AL (1998): Erectile dysfunction: apractical approach for primary care. Geriatrics $\underline{53}, 34-48$

Corney RH (1990): Sex differences in gneral practice attendance and help seeking for minor illness. J Psychosom Res 34, 525 - 534

Dahmer J: Anamnese und Befund. 7. Auflage; Thieme, Stuttgart 1994

Dunn KM, Croft PR, Hackett GI (1998): Sexual problems: a study of the prevalence and need for health care in the general population. Fam Pract $\underline{15}, 519-524$

Dunn KM, Croft PR, Hackett GI (1999): Association of sexual problems with social, psychological and physical problems in men and women: a cross sectional population survey. J Epidemiol Community Health $\underline{53}, 144-148$

Ende J, Rockwell S, Glasgow M (1984): The sexual history in general medicine practice. Arch Intern Med 144, 558-561

Ewert W: Der Patient im Kontext der Familie; in: Allgemeinmedizin und Familienmedizin. 2. Auflage; hrsg. v. Kochen MM; Hippokrates, Stuttgart 1998, 36 - 46

Feldman HA, Goldstein I, Hatzichristou DG, Krane RJ, McKinlay JB (1994): Impotence and its medical and psychosocial correlates: Results of the Massachusetts Male Aging Study. J Urol 151, 54 - 61

Finger WW, Lund M, Slagle MA (1997): Medications that may contribute to sexual disorders: A guide to assessment and treatment in general practice. J Fam Pract $\underline{44}, 33-43$

Foreman MM, Doherty PC: Experimental approaches for the development of pharmacological therapies for erectile dysfunction; in: Sexual Pharmacology; hrsg. v. Riley AJ u. a.; Clarendon Press, Oxford 1993, 87 - 113 
Goldstein I, Lue TF, Padma-Nathan H, Rosen RC, Steers WD, Wicker PA (1998): Oral Sidenafil in the treatment of erectile dysfunction. N Engl J Med $\underline{338}, 1397$ 1404

Guirguis WR, (1998): Oral treatment of erectile dysfunction: from herbal remedies to designer drugs. J Sex Marital Ther 24, $69-73$

Halvorsen JG, Metz ME (1991): Sexual dysfunction, part I: Classification, etiology and pathogenesis. J Am Board Fam Pract $\underline{5}, 51-61$

Hartmann U (1998): Erektile Dysfunktionen: Psychologische Aspekte in Verursachung, Diagnostik und Therapie. Ther Umsch 55, $352-356$

Hengeveld MW (1998): Somatization of erectile disorders by the introduction of sildenafil. Ned Tijdschr Geneeskd 노, 2333-2336

Himmel W (2000): Selbsmedikation - ökonomische, sozialpharmakologische und toxikologische Aspekte. Dtsch Med Wochenschr 125, im Druck

Himmel W, Kochen MM (1998): Der familienmedizinische Ansatz in der Allgemeinmedizin. Dtsch Ärztebl 95, $1794-1797$

Holzapfel S (1993): Sexual medicine in general practice. Can Fam Physician $\underline{39}$, $608-621$

Ittner E, Himmel W, Kochen MM (1997a): Management of involuntary childlessness in practice - patients' and physicians' views. Br J Gen Pract 47, 105- 106

Ittner E, Himmel W, Kochen MM (1997b): Gehört das Problem „ungewollte Kinderlosigkeit" zum familienmedizinischen Auftrag des Hausarztes? Psychother Psychosom Med Psychol $\underline{49}, 263-270$ 
Kerek-Bodden $\mathrm{H}$, Zentralinstitut für kassenärztliche Versorgung, Köln: persönliche Mitteilung, 2000

Kerek-Bodden H, Koch H, Brenner G, Flatten G (2000): Diagnosespektrum und Behandlungsaufwand des allgemeinärztlichen Patientenklientels. Z Arztl Fortbild Qualitatssich $\underline{94}, 21-30$

Kloner RA, Jarow JP (1999): Erectile dysfunction and sildenafil citrate and cardiologists. Am J Cardiol $\underline{83}, 576-582$

Kochen MM (2000): The best prescription may be no prescription (Editorial). Dtsch Med Wochenschr 125, im Druck

Kockott G: Männliche Sexualität. Hippokrates Verlag, Stuttgart 1988

Kockott G (1989): Die Psyche ist immer dabei! Sexualmedizin 18, 294 - 299

Kuritzky L, Ahmed O, Kosch S (1998): Management of impotence in primary care. Compr Ther 24, $137-146$

Laumann EO, Paik A, Rosen RC (1999): Sexual dysfunction in the United States. Prevalence and predictors. JAMA 281, $537-544$

Levine SB (1992): Male sexual problems and the general physician. J Med Assoc Ga $\underline{81}, 211-215$

Loewit K (1996): Sexualprobleme - Beratungsmöglichkeiten beim praktischen Arzt. Fortschr Med 30, 387 - 392

McCabe MP (1997): Intimacy and quality of life among sexually dysfunctional men and women. J Sex Marital Ther $\underline{23}, 276-290$

Merrill JM, Laux LF, Thornby JI (1990): Why doctors have difficulty whith sex histories. South Med J $\underline{83}, 613-617$ 
Metz ME, Seifert MH (1990): Men's expectations of physicians in sexual health concerns. J Sex Marital Ther $\underline{16}, 79-88$

Miller TA (2000): Diagnostic evaluation of erectile dysfunction. Am Fam Physician $\underline{61}, 95-104$

Mobley DF, Baum N (1998): When patients request the impotence pill. Postgrad Med 104, 55-66

Moore JT, Goldstein Y (1980): Sexual problems among family medicine patients. J Fam Pract $\underline{10}, 243-247$

Morales A, Gingell C, Collins M, Wicker PA, Osterloh ICH (1998): Clinical safety of oral sildenafil citrate (VIAGRA $\otimes$ ) in the treatment of erectile dysfunction. Int $\mathrm{J}$ Impot Res $\underline{10}, 69-73$

Müller-Oerlinghausen B, Ringel I, Munter KH (1999): The relevance of psychotropics-induced sexual dysfunction within the ADR voluntary reporting system in Germany. Eur J Clin Pharmacol 55, 577 - 581

Nehra A, Barrett DM, Moreland RB (1999): Pharmacotherapeutic advances in the treatment of erectile dysfunction. Mayo Clin Proc $\underline{74}, 709-721$

Perttula E (1999): Physician attitudes and behaviour regarding erectile dysfunction in at-risk patients from rural community. Postgrad Med J $\underline{75}, 83-85$

Pinnock CB, Stapleton AM, Marshall VR (1999): Erectile dysfunction in the community: a prevalence study. Med J Aust $\underline{171}, 353-357$

Porst $\mathrm{H}$ : Was jedermann über Sexualität und Potenz wissen sollte. TRIAS, Stuttgart 1991

Porst H (1992): Erektile Dysfunktion: Mögliche Ursachen - individuelle Heilungschancen. Pro Familia Mag 20, 10 - 13 
Pschyrembel: Klinisches Wörterbuch. 258. Auflage; Walter de Gruyter Verlag, Berlin 1998

Rakel M: The family physician; in: Textbook of family practice. 5. Aufl; hrsg. v. Rakel M; Saunders, Philadelphia 1995, 3 - 19

Read S, King M, Watson J (1997): Sexual dysfunction in primary medical care: prevalence, characteristics and detection by the general practitioner. J Public Health Med $\underline{19}, 287-391$

Riley AJ, Athanasiadis L (1997): Impotence and its non - surgical management. $\mathrm{Br}$ $\mathrm{J}$ Clin Pract $\underline{\text { 11, }} 99$ - 105

Rönsberg W: Sexualberatung; in: Allgemeinmedizin und Familienmedizin. 2. Auflage; hrsg. v. Kochen MM; Hippokrates Verlag, Stuttgart 1998, 245 - 254

Sanders D (1993): Talking about sex. Practitioner 237, $674-679$

SAS Institute Inc.: SAS/SAT User's Guide, Version 6, Fourth Edition; SAS Institute Inc., Cary/NC 1989

Schaudig A (1998): Beratungsproblem Erektile Dysfunktion. Allgemeinarzt 17, 1625 $-1642$

Schaudig A (1999): Erektile Dysfunktion: Update Februar 1999. Allgemeinarzt $\underline{18}$, $280-283$

Schilling G, Müller MJ, Haidl G (1999): Sexuelle Unzufriedenheit und Körperbeschwerden bei männlicher Infertilität. Psychother Psychosom Med Psychol $\underline{49}, 256$ $-263$

Senger G, Hoffmann W (1996): Lädierte Männlichkeit. Weibliche Emanzipation und männliche Potenz. Sexualmedizin $\underline{17}, 74-78$ 
Shabsigh R, Klein LT, Seidman S, Kaplan SA, Lehrhoff BJ, Ritter JS (1998): Increased incidence of depressive symptoms in men with erectile dysfunction. Urology $\underline{52}$, $848-852$

Shahar E, Lederer J, Herz MJ (1991): The use of a self-report-questionnaire to assess the frequency of sexual dysfunction in family practice Clinics. Fam Pract $\underline{8}, 206$ $-212$

Sigusch V (1995): Organotherapien bei sexuellen Funktionsstörungen: Eine Übersicht. Z Sexualforsch $\underline{8}, 329-352$

Suter S (1998): Erektile Dysfunktion: Abklärung und Behandlung beim Hausarzt was ist sinnvoll? Ther Umsch $\underline{55}, 357-360$

Tiefer L (1993): Über die fortschreitende Medikalisierung männlicher Sexualität. Z Sexualforsch $\underline{6}, 119-131$

Tiefer L (1998): Doing the Viagra tango. Z Sexualforsch 11, 346-352

Tiefer L, Schütz - Müller D (1995): Psychological issues in diagnosis and treatment of erectile disorders. Urol Clin North Am 22, 767 - 773

Tudiver F, Talbot Y (1999): Why don't men seek help? Family physicians' perspectives on help-seeking behavior in men. J Fam Pract $\underline{48}, 47-52$

Utiger RD (1998): A pill for impotence. N Engl J Med 338, 1458 - 1459 


\section{Danksagung}

Ich danke Herrn Prof. Dr. Kochen, MPH, FRCGP, für die freundliche Überlassung des Themas der Dissertation.

Frau Dr. Ittner danke ich für die engagierte und kontinuierliche Betreuung meiner Arbeit sowie Herrn Priv.-Doz. Dr. Himmel für die konstruktive Kritik.

Mein besonderer Dank gilt den Ärzten und Arzthelferinnen in den an der Studie beteiligten Praxen.

Schließlich danke ich den Patienten, die sich bereit erklärten, an der Untersuchung teilzunehmen. 


\section{Lebenslauf}

Am 06.08.1972 wurde ich in Lingen (Ems) geboren.

1979 bis 1983 besuchte ich die Grundschule in Lingen (Ems). In den folgenden zwei Jahren war ich Schülerin der Orientierungsstufe und wechselte danach auf das Gymnasium Georgianum derselben Stadt. Dort erwarb ich am 11.05.1993 die allgemeine Hochschulreife. Das Schuljahr 1989/1990 verbrachte ich in Troyes (Frankreich).

Im Sommersemester 1995 nahm ich mein Studium der Humanmedizin in Göttingen auf. Nach bestandener Ärztlicher Vorprüfung am 14.03.1997 und bestandenem ersten Abschnitt der Ärztlichen Prüfung am 24.03.1998 befinde ich mich zur Zeit im 10. Fachsemester und beabsichtige, den zweiten Abschnitt der Ärztlichen Prüfung im Herbst 2001, den dritten Abschnitt der Ärztlichen Prüfung entsprechend ein Jahr später zu absolvieren.

Ich habe zwei Kinder, Bartolomèo und Ernesto Aschka, die 1992 bzw. 1994 geboren wurden. Mit meinem Lebensgefährten, Herbert Masslau, lebe ich seit der Geburt unseres ersten Sohnes zusammen. 